Estudo de uma classe de equações elípticas via métodos variacionais e topológicos 



\title{
Estudo de uma classe de equações elípticas via métodos variacionais e topológicos
}

\author{
Júlia Silva Silveira Borges
}

Orientadora: Prof. Dr. Sérgio Henrique Monari Soares

Dissertação apresentada ao Instituto de Ciências Matemáticas e de Computação - ICMC-USP, como parte dos requisitos para obtenção do título de Mestre em Ciências - Matemática . VERSÃO REVISADA

USP - São Carlos

Junho de 2012 
Ficha catalográfica elaborada pela Biblioteca Prof. Achille Bassi e Seção Técnica de Informática, ICMC/USP, com os dados fornecidos pelo(a) autor(a)

Silva Silveira Borges, Júlia
Este
métodos variacionais e topológicos / Júlia Silva
Silveira Borges; orientador Sérgio Henrique Monari
Soares. - São Carlos, 2012.
68 p.
Dissertação (Mestrado - Programa de Pós-Graduação en
Matemática)-- Instituto de Ciências Matemáticas e
de Computação, Universidade de São Paulo, 2012.
1. Equações diferenciais parciais. 2. Equações
diferenciais parciais elipticas. 3. Problemas
elípticos assintoticamente lineares. I. Monari
Soares, Sérgio Henrique, orient. II. Título.


Aos meus pais

Silvia e Edison. 



\section{Agradecimentos}

Agradeço primeiramente a Deus por ter permitido que eu pudesse realizar os meus sonhos. Por sempre me mostrar das mais diversas maneiras porque estou aqui e que caminho devo seguir.

Aos meus pais, Sílvia e Edison, e a minha irmã, Lívia, agradeço pelo amor incondicional, pela ajuda e determinação em sonhar junto e realizar junto. Agradeço pela esperança que sempre me deram, confiança que em mim depositaram e certeza de que eu poderia chegar até aqui.

Ao meu marido Rodrigo, que soube esperar e caminhar junto, agradeço todo apoio, dedicação e luta.

Aos amigos, agradeço àqueles a quem tive que deixar em Franca e souberam compreender, e àqueles que encontrei no meio do caminho.

Aos professores da USP, tão importantes em minha formação, agradeço o quanto ensinaram e ajudaram a realizar. Em especial ao Prof. Wagner e a Profa. Sandra pelos conselhos e confiança.

Ao meu orientador Prof. Sérgio Monari, agradeço por tantas horas de trabalho trasmitindo-me sua experiência em forma de ajuda, orientação e conselhos.

E por último, agradeço a CAPES pelo apoio financeiro sem o qual não seria possível a realização deste trabalho. 

"Feliz aquele que transfere o que sabe e aprende o que ensina." Cora Coralina 



\section{Resumo}

Alguns problemas elípticos assintoticamente lineares são considerados e é provada a existência de solução. Os principais resultados são estabelecidos de dois modos distintos e as provas são baseadas em resultados clássicos da teoria de pontos críticos, a saber: minimização, princípio variacional de Ekeland, grau topológico, teorema do ponto de sela e o teorema do passo da montanha. 

Some asymptotically linear elliptic problems are considered and solutions are proved to exist. The main results are proved in two different ways. The proofs rely on some classical results in Critical Point Theory such as minimization, Ekeland variational principle, topological degree, saddle point theorem and mountain pass theorem. 

Resumo i

$\begin{array}{lll}\text { Abstract } & \text { iii }\end{array}$

Introdução

1 Teorema 0.0.1 5

1.1 Demonstração via minimização. . . . . . . . . . . . . . . . . . . . . 5

1.1.1 Demonstração do Teorema 0.0.1. . . . . . . . . . . . . . . . 6

1.2 Demonstração via o Princípio Variacional de Ekeland . . . . . . . . . . . . . . 9

1.2.1 Princípio Variacional de Ekeland . . . . . . . . . . . . . . . . . 9

1.2.2 A Condição de Palais-Smale . . . . . . . . . . . . . . . . . . . . . . . . 10

1.2 .3 Demonstração do Teorema $0.0 .1 \ldots \ldots$. . . . . . . . . . . 16

$\begin{array}{lll}2 & \text { Teorema } 0.0 .2 & 17\end{array}$

2.1 Demonstração via teoria do grau . . . . . . . . . . . . . . . . . . . . 17

2.1.1 Estimativa a Priori . . . . . . . . . . . . . . . . . . 17

2.1 .2 Demonstração do Teorema $0.0 .2 \ldots \ldots \ldots$. . . . . . . . . . 18

2.2 Demonstração via o teorema do ponto de sela . . . . . . . . . . . . . . . . 20

2.2 .1 Lema de Deformação . . . . . . . . . . . . . . . . . . . . . . . . 20

2.2 .2 Teorema do Ponto de Sela . . . . . . . . . . . . . . . . . . . . . 23

2.2 .3 Demonstração do Teorema $0.0 .2 \ldots \ldots$. . . . . . . . . . . . 24

$\begin{array}{llr}3 & \text { Teorema } 0.0 .3 & 27\end{array}$

3.1 Teorema do Passo da Montanha . . . . . . . . . . . . . . . . . . . . . . 27

3.2 Demonstração do Teorema $0.0 .3 \ldots \ldots \ldots \ldots$. . . . . . . . . . . . 28 
A.1 Teoremas de Imersão . . . . . . . . . . . . . . . . . . . . . . . . . . . . . . . . 34

B Alguns Resultados $\quad 35$

$\begin{array}{lll}\text { C O Funcional Energia } & 37\end{array}$

D Espectro do Laplaciano $\quad 45$

D.1 O operador solução . . . . . . . . . . . . . . . . . . . . . . . 45

D.2 Propriedades do operador solução . . . . . . . . . . . . . . . . . . 46

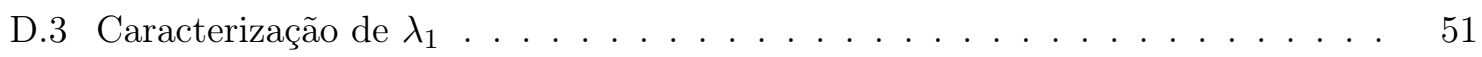

E Alguns resultados sobre Teoria do Grau

E.1 Grau de Brouwer . . . . . . . . . . . . . . . . . . . . . . 55

E.1.1 Caso Regular . . . . . . . . . . . . . . . . . . 55

E.1.2 Definição do Grau para $\varphi \in \mathcal{C}^{2}(\bar{\Omega})$ e $b \notin \varphi(\partial \Omega) \ldots \ldots . . . . . . .556$

E.1.3 Definição de Grau de Brouwer para funções contínuas. . . . . . . . . 56

E.1.4 Principais propriedades do Grau de Brouwer: . . . . . . . . . . . . 57

E.1.5 Consequências das Propriedades Principais . . . . . . . . . . . 57

E.1.6 Índice de Brouwer . . . . . . . . . . . . . . . . . . . . . 58

E.2 O Grau de Leray-Schauder . . . . . . . . . . . . . . . . . . 59

E.2.1 Principais Propriedades do Grau de Leray-Schauder . . . . . . . . . . 62

E.2.2 Consequência das Propriedades Principais . . . . . . . . . . . . . . 62

E.2.3 Índice de Leray-Schauder . . . . . . . . . . . . . . . . . . . . . 63

$\begin{array}{ll}\text { Referências Bibliográficas } & 67\end{array}$ 


\section{Introdução}

Neste trabalho propomos o estudo de alguns problemas em equações diferenciais parciais elípticas semilineares. O objetivo aqui não é a generalidade e nem a atualidade dos resultados, mas sim a aplicação de diferentes métodos da teoria de pontos críticos, tais como minimização, princípio variacional de Ekeland, teorema do ponto de sela, teorema do passo da montanha e o grau topológico. Neste sentido, os problemas em equações diferenciais escolhidos como objeto de estudo são aqueles que permitem a utilização de diferentes métodos de resolução. O modelo típico a ser considerado é o problema

$$
\left\{\begin{array}{l}
-\Delta u=f(x, u), \quad x \in \Omega, \\
u=0, \quad x \in \partial \Omega,
\end{array}\right.
$$

onde $\Omega \subset \mathbb{R}^{N}, N \geq 3$, é um domínio limitado com fronteira $\partial \Omega$ suave e $f \in \mathcal{C}(\bar{\Omega} \times \mathbb{R}, \mathbb{R})$. Consideraremos problemas assintoticamente lineares, ou seja, quando os limites abaixo existem e são funções de $L^{\infty}(\Omega)$ :

$$
\lim _{s \rightarrow \infty} \frac{f(x, s)}{s}=a(x) \quad \text { e } \lim _{s \rightarrow-\infty} \frac{f(x, s)}{s}=b(x) .
$$

Com o intuito de ilustrar os vários métodos de resolução, vamos estudar as demonstrações dos seguintes resultados:

Teorema 0.0.1. Suponha que $f \in \mathcal{C}(\bar{\Omega} \times \mathbb{R}, \mathbb{R})$ e satisfaz

$$
a(x), b(x) \leq \mu<\lambda_{1} \text {, quase sempre em } \Omega,
$$

onde $\mu$ é uma constante e $\lambda_{1}$ é o primeiro autovalor do Laplaciano com condição de Dirichlet. Então o problema (1) possui uma solução clássica.

Teorema 0.0.2. Suponha que $f \in \mathcal{C}(\bar{\Omega} \times \mathbb{R}, \mathbb{R})$ é Lipschitziana em relação à segunda variável e satisfaz

$$
\lambda_{j}<c_{j} \leq a(x), b(x) \leq c_{j+1}<\lambda_{j+1} \text {, quase sempre em } \Omega,
$$


onde $c_{j}$ e $c_{j+1}$ são constantes e $\lambda_{j}, j \in \mathbb{N}$ são os autovalores do Laplaciano com condição de Dirichlet. Então o problema (1) possui uma solução clássica.

Por solução do problema (1), entende-se uma solução fraca, ou seja, uma função $u$ no espaço de Sobolev $H_{0}^{1}(\Omega)$ satisfazendo a equação

$$
\int_{\Omega} \nabla u \nabla \varphi d x=\int_{\Omega} f \varphi d x, \forall \varphi \in H_{0}^{1}(\Omega) .
$$

Em busca de soluções fracas do problema (1) definimos o funcional energia $\phi: H_{0}^{1}(\Omega) \rightarrow \mathbb{R}$ por

$$
\phi(u)=\frac{1}{2} \int_{\Omega}|\nabla u|^{2} d x-\int_{\Omega} F(x, u) d x, \text { onde } F(x, u)=\int_{0}^{u} f(x, s) d s .
$$

Sob a hipótese (2) e que $f$ é é localmente Lipschitziana, é provado no Apêndice deste trabalho que o funcional $\phi \in \mathcal{C}^{1}\left(H_{0}^{1}(\Omega), \mathbb{R}\right)$ e que

$$
\phi^{\prime}(u) \varphi=\int_{\Omega}(\nabla u \nabla \varphi-f(x, u) \varphi) d x, \quad \forall \varphi \in H_{0}^{1}(\Omega) .
$$

Um ponto crítico de $\phi$ é uma função $u \in H_{0}^{1}(\Omega)$ tal que $\phi^{\prime}(u)=0$, isto é, $\phi^{\prime}(u) \varphi=0$ para todo $\varphi \in H_{0}^{1}(\Omega)$. Portanto, $u$ é solução fraca do problema (1) se, e somente se, $u$ é ponto crítico do funcional $\phi$. Sendo assim, os teoremas acima serão provados utilizando alguns resultados da teoria de pontos críticos. Utilizando a teoria de regularidade em equações elípticas, podemos concluir que as soluções fracas são também soluções clássicas, ou seja, funções em $\mathcal{C}^{2}(\Omega, \mathbb{R}) \cap \mathcal{C}(\bar{\Omega}, \mathbb{R})$ satisfazendo a equação $(1)$.

As demonstrações desses teoremas são feitas por dois métodos: topológico e variacional. O Teorema 0.0.1 será demonstrado de duas maneiras distintas: uma por minimização e a outra utilizando o princípio variacional de Ekeland, o que são os objetos do Capítulo 1. No Capítulo 2, utilizamos o grau topológico e o teorema do ponto de sela para apresentar duas demonstrações diferentes do Teorema 0.0.2.

Por fim, no Capítulo 3, seguindo o artigo [12], faremos uso do teorema do passo da montanha para provar a existência de uma solução para uma classe de problemas assintoticamente lineares. Mais precisamente, apresentaremos uma demonstração do seguinte resultado:

Teorema 0.0.3. Seja $f: \mathbb{R} \rightarrow \mathbb{R}$ uma função diferenciável tal que $f(0)=0$,

$$
f^{\prime}(0)<\lambda_{1}
$$

e

Então, o problema

$$
\lambda_{1}<\lim _{u \rightarrow \infty} \frac{f(u)}{u}<\infty .
$$

$$
\left\{\begin{array}{l}
-\Delta u=f(u), \quad x \in \Omega, \\
u>0, \quad x \in \Omega, \\
u=0, \quad x \in \partial \Omega,
\end{array}\right.
$$

possui pelo menos uma solução. 
Para citar um exemplo, podemos considerar

$$
f(x, s)=f(s)=\frac{s^{3}}{1+\alpha s^{2}}, \text { para } s \in \mathbb{R}, \text { onde } \alpha>0 .
$$

Mediante escolhas apropriadas de $\alpha$, a função f satisfaz as respectivas hipóteses dos teoremas anteriores.

Observação 0.0.1. Os resultados citados também são válidos para os casos em que $N=$ 1 ou 2. As demonstrações dos resultados para $N=1$ são as mesmas, enquanto que as respectivas demonstrações quando $N=2$ requerem o uso de outros argumentos. Para facilitar a discussão dos resultados, vamos nos concentrar no caso $N \geq 3$. 



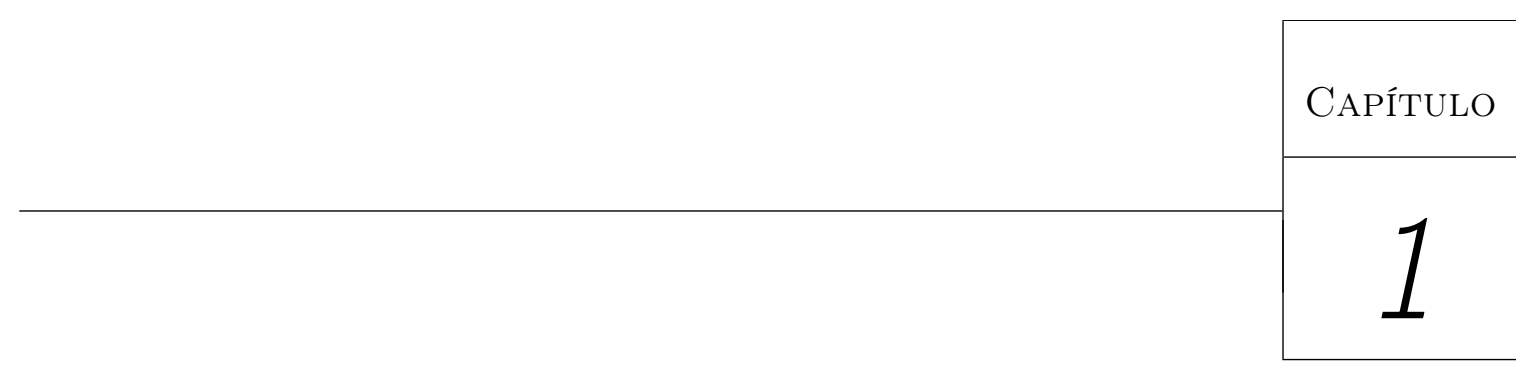

Teorema 0.0.1

Este capítulo é dedicado a demonstrar o Teorema 0.0.1 por meio de duas variações do método de minimização e a apresentação dar-se-á nas seções a seguir.

\subsection{Demonstração via minimização.}

A demonstração é consequência do teorema mostrado abaixo que nos garante que o ínfimo do funcional $\phi$ é atingido.

Definição 1.1.1. Seja $X$ um espaço topológico. Um funcional $\phi: X \rightarrow \mathbb{R} \cup\{\infty\}$ será dito semicontinuo inferiormente se para todo $a \in \mathbb{R}$, o conjunto $\{x \in X ; \phi(x)>a\}$ for aberto.

Teorema 1.1.1. Sejam $X$ um espaço topológico compacto e $\phi: X \rightarrow \mathbb{R}$ uma função semicontinua inferiormente. Então $\phi$ é limitada inferiormente e existe $u \in X$ tal que

$$
\phi(u)=\inf _{X} \phi
$$

\section{Demonstração:}

Definamos os conjuntos $A_{n}=\{x \in X ; \phi(x)>-n\}$ para todo $n \in \mathbb{N}$. Como $\phi$ é semicontínuo inferiormente, os conjuntos $A_{n}$ são abertos. Além disso, $A_{n} \subset A_{n+1}$. Assim, $\left\{A_{n}\right\}_{n \in \mathbb{N}}$ é uma cobertura aberta de $X$. Sendo $X$ compacto, existe um $n_{0} \in \mathbb{N}$ tal que

$$
X \subset \bigcup_{j=1}^{n_{0}} A_{j} .
$$


Deste modo, $\phi(x)>-n_{0}, \forall x \in X$, o que implica que $\phi$ é limitada inferiormente. Seja agora $l=\inf \{\phi(x) ; x \in X\}$. Suponha, por absurdo, que $l$ não é atingido, isto é,

não existe $x \in X$ tal que $\phi(x)=l$.

Assim,

$$
X=\bigcup_{n=1}^{\infty}\left\{x \in X ; \phi(x)>l+\frac{1}{n}\right\} .
$$

E, como os conjuntos

$$
\left\{x \in X ; \phi(x)>l+\frac{1}{n}\right\}
$$

são abertos para todo $n \in \mathbb{N}$, temos que

$$
\left\{x \in X ; \phi(x)>l+\frac{1}{n}\right\}_{n \in \mathbb{N}}
$$

é uma cobertura aberta de $X$. Novamente pela compacidade de $X$, existe $n_{1} \in \mathbb{N}$ tal que

$$
X \subset \bigcup_{n=1}^{n_{1}}\left\{x \in X ; \phi(x)>l+\frac{1}{n}\right\} .
$$

Logo,

$$
\phi(x)>l+\frac{1}{n_{1}}, \forall x \in X,
$$

o que contradiz o fato de $l$ ser o ínfimo. Portanto, existe $x_{0} \in X$ tal que

$$
\phi\left(x_{0}\right)=\inf _{X} \phi .
$$

\subsubsection{Demonstração do Teorema 0.0.1.}

Primeiramente observemos que pela condição (2), existem constantes $c>0$ e $\tilde{\mu}$, com $\tilde{\mu}<\lambda_{1}$ e $\tilde{\mu} \geq \mu$, tais que

$$
f(x, s) \leq \tilde{\mu}|s|+c, \forall s \in \mathbb{R}, \forall x \in \Omega .
$$

De fato, por (2), temos

$$
a(x), b(x) \leq \mu<\lambda_{1} .
$$

Como $f$ é assintoticamente linear, pelas equações (2) temos que dado $\varepsilon>0$, existem $s_{0}, s_{1} \in \mathbb{R}$ tais que

$$
\left|\frac{f(x, s)}{s}-a(x)\right| \leq \varepsilon, \forall s \geq s_{1}
$$

e

$$
\left|\frac{f(x, s)}{s}-b(x)\right| \leq \varepsilon, \forall s \leq s_{0} .
$$


Assim,

$$
\left|\frac{f(x, s)}{s}\right|-|a(x)| \leq\left|\frac{f(x, s)}{s}-a(x)\right| \leq \varepsilon, \forall s \geq s_{1} .
$$

Logo,

$$
\left|\frac{f(x, s)}{s}\right| \leq \varepsilon+|a(x)|,
$$

o que implica que

$$
|f(x, s)| \leq \varepsilon|s|+a(x)|s|=(\varepsilon+\mu)|s|=\tilde{\mu}|s|, \forall s \geq s_{1} .
$$

Tomemos $\varepsilon>0$ de modo que $\tilde{\mu}=\varepsilon+\mu<\lambda_{1}$. Analogamente, temos:

$$
|f(x, s)| \leq \tilde{\mu}|s|, \forall s \leq s_{0} .
$$

E, assim,

$$
|f(x, s)| \leq \tilde{\mu}|s|, \forall s \leq s_{0} \text { e } s \geq s_{1} .
$$

Agora note que como $f$ é localmente Lipschtziana, existe $c>0$ tal que

$$
|f(x, s)| \leq c, \forall s \text { satisfazendo } s_{0} \leq s \leq s_{1} .
$$

Assim, existe $\tilde{\mu}$, com $\tilde{\mu}<\lambda_{1}$ e $\tilde{\mu} \geq \mu$ tal que

$$
|f(x, s)| \leq \tilde{\mu}|s|+c, \forall s \in \mathbb{R}, \forall x \in \Omega .
$$

Assim, para $F(x, s)=\int_{0}^{s} f(x, t) d t$, temos

$$
F(x, s) \leq \frac{\hat{\mu}}{2} s^{2}+C, \forall s \in \mathbb{R}, \forall x \in \Omega,
$$

onde $\tilde{\mu} \leq \hat{\mu}<\lambda_{1}$, para alguma constante $C>0$. Consequentemente,

$$
\phi(u)=\frac{1}{2} \int_{\Omega}|\nabla u|^{2} d x-\int_{\Omega} F(x, u) d x \geq \frac{1}{2} \int_{\Omega}|\nabla u|^{2} d x-\frac{\hat{\mu}}{2} \int_{\Omega} u^{2} d x-C .
$$

Pela desigualdade de Poincaré,

$$
\phi(u) \geq \frac{1}{2} \int_{\Omega}|\nabla u|^{2} d x-\frac{\hat{\mu}}{2 \lambda_{1}} \int_{\Omega}|\nabla u|^{2} d x-C .
$$

Deste modo, obtemos

$$
\phi(u) \geq \frac{1}{2}\left(1-\frac{\hat{\mu}}{\lambda_{1}}\right) \int_{\Omega}|\nabla u|^{2} d x-C .
$$

A equação (1.2) nos diz que $\phi: H_{0}^{1}(\Omega) \rightarrow \mathbb{R}$ é limitada inferiormente. Também que $\phi$ é coerciva, isto é,

$$
\phi(u) \rightarrow \infty \text { quando }\|u\|_{H_{0}^{1}(\Omega)} \rightarrow \infty .
$$

Tomemos agora $R>0$ tal que

$$
\phi(u) \geq 1, \forall u \in H_{0}^{1}(\Omega),\|u\|_{H_{0}^{1}(\Omega)} \geq R,
$$


o que segue de (1.2), isto é, o funcional $\phi$ é limitado inferiormente no complementar de uma bola.

Consideremos o funcional $\phi$ restrito à bola fechada $B_{R}[0]$. No Apêndice $\mathrm{C}$, mostramos que o funcional $J: H_{0}^{1}(\Omega) \rightarrow \mathbb{R}$ definido por

$$
J(u)=\int_{\Omega} F(x, u) d x
$$

é sequencialmente fracamente contínuo. Sendo $B_{R}[0]$ metrizável, J é fracamente contínuo em $B_{R}[0]$. Usando o fato que a norma $\|\cdot\|_{H_{0}^{1}(\Omega)}$ é fracamente semicontínua inferiormente, segue que o funcional

$$
\phi(u)=\frac{1}{2} \int_{\Omega}|\nabla u|^{2} d x-J(u)
$$

também é fracamente semicontínuo inferiormente na bola $B_{R}[0]$. Logo, pelo Teorema 1.1.1, existe $u_{0} \in B_{R}[0]$ tal que

$$
\phi\left(u_{0}\right)=\inf _{B_{R}[0]} \phi .
$$

Como $\phi(0)=0$, segue que $\phi\left(u_{0}\right) \leq 0$. Deste modo, $\left\|u_{0}\right\|_{H_{0}^{1}(\Omega)}<R$, pois $\phi(u) \geq 1$ para todo $u \in H_{0}^{1}(\Omega)$ com $\|u\|_{H_{0}^{1}(\Omega)} \geq R$. Consequentemente, existe $\varepsilon>0$ tal que

$$
\left\|u_{0}+t w\right\|_{H_{0}^{1}(\Omega)}<R, \forall 0<t \leq \varepsilon,\|w\|_{H_{0}^{1}(\Omega)}=1 .
$$

De fato, uma vez que $\left\|u_{0}\right\|_{H_{0}^{1}(\Omega)}<R$, tomemos $\varepsilon>0$ tal que $\|u\|_{H_{0}^{1}(\Omega)}+\varepsilon<R$. Deste modo,

$$
\begin{aligned}
\left\|u_{0}+t w\right\|_{H_{0}^{1}(\Omega)} & \leq\left\|u_{0}\right\|_{H_{0}^{1}(\Omega)}+t\|w\|_{H_{0}^{1}(\Omega)} \\
& \leq\left\|u_{0}\right\|_{H_{0}^{1}(\Omega)}+\varepsilon<R,
\end{aligned}
$$

para todo $0<t \leq \varepsilon$ e $\|w\|_{H_{0}^{1}(\Omega)}=1$. Como $\phi\left(u_{0}\right)=\inf \left\{\phi(u) ; u \in B_{R}[0]\right\}$, segue que

$$
\phi\left(u_{0}\right) \leq \phi\left(u_{0}+t w\right) .
$$

E, daí,

$$
\frac{\phi\left(u_{0}+t w\right)-\phi\left(u_{0}\right)}{t} \geq 0, \quad \forall 0<t \leq \varepsilon .
$$

Tomando o limite quando $t \rightarrow 0^{+}$, obtemos:

$$
\phi^{\prime}\left(u_{0}\right) w \geq 0, \forall w \in H_{0}^{1}(\Omega),\|w\|_{H_{0}^{1}(\Omega)}=1 .
$$

Seja agora $v \in H_{0}^{1}(\Omega), v \neq 0$ e consideremos

$$
w=\frac{v}{\|v\|_{H_{0}^{1}(\Omega)}} .
$$

Então

$$
\begin{aligned}
\phi^{\prime}\left(u_{0}\right) w & =\int_{\Omega} \nabla u_{0} \nabla\left(\frac{v}{\|v\|_{H_{0}^{1}(\Omega)}}\right)-\int_{\Omega} f\left(x, u_{0}\right)\left(\frac{v}{\|v\|_{H_{0}^{1}(\Omega)}}\right) \\
& =\frac{1}{\|v\|_{H_{0}^{1}(\Omega)}}\left(\int_{\Omega} \nabla u_{0} \nabla v-\int_{\Omega} f\left(x, u_{0}\right) v\right) .
\end{aligned}
$$


Como $\phi^{\prime}\left(u_{0}\right) w \geq 0$ e $\|v\|_{H_{0}^{1}(\Omega)}>0$, segue que

$$
\phi^{\prime}\left(u_{0}\right) v \geq 0, \forall v \in H_{0}^{1}(\Omega) .
$$

Por outro lado, sendo $w \in H_{0}^{1}(\Omega)$, temos $-w \in H_{0}^{1}(\Omega)$. Deste modo,

$$
0 \leq \phi^{\prime}\left(u_{0}\right)(-w)=-\phi^{\prime}\left(u_{0}\right) w \leq 0,
$$

uma vez que $\phi^{\prime}\left(u_{0}\right) w \geq 0$. Logo,

$$
\phi^{\prime}\left(u_{0}\right) w=0, \forall w \in H_{0}^{1}(\Omega),
$$

o que implica que $\phi^{\prime}\left(u_{0}\right)=0$. Portanto, $u_{0}$ é um ponto crítico de $\phi$, e assim, é uma solução fraca do problema (1).

\subsection{Demonstração via o Princípio Variacional de Ekeland}

Nesta seção demonstraremos o Teorema 0.0.1 utilizando um método variacional e nossa ferramenta para demonstração será o Princípio Variacional de Ekeland. Além disso, precisaremos mostrar que o funcional $\phi$ em determinadas condições, satisfaz a condição (PS) que será descrita no segundo item desta seção.

\subsubsection{Princípio Variacional de Ekeland}

Teorema 1.2.1. Sejam $X$ um espaço métrico completo e $\phi: X \rightarrow \mathbb{R}$ um funcional semicontínuo inferiormente e limitado inferiormente. Sejam $\varepsilon>0$ e $u \in X$ dados, tais que

$$
\phi(u) \leq \inf _{X} \phi+\varepsilon
$$

Então existe $v \in X$ tal que

$$
\begin{gathered}
\phi(v) \leq \phi(u), \\
d(u, v) \leq 1
\end{gathered}
$$

e, para cada $w \neq v$ em $X$,

$$
\phi(w)>\phi(v)-\varepsilon d(v, w) .
$$

\section{Demonstração:}

Definamos a seguinte relação em $X$ :

$$
w \leq v \Leftrightarrow \phi(w)+\varepsilon d(v, w) \leq \phi(v) .
$$

Tal relação define uma ordem em $X$. Construiremos indutivamente uma sequência $\left\{u_{n}\right\}_{n}$. Seja $u_{0}=u$ e suponhamos que $u_{n}$ é conhecida. Defina $S_{n}=\left\{w \in X ; w \leq u_{n}\right\}$ e escolha $u_{n+1} \in S_{n}$ tal que

$$
\phi\left(u_{n+1}\right) \leq \inf _{S_{n}} \phi+\frac{1}{n+1}
$$


Como $u_{n+1} \leq u_{n}$ temos que $S_{n+1} \subset S_{n}$. Além disso, como $\phi$ é semicontínua inferiormente, $S_{n}$ é fechado. Seja agora $w \in S_{n+1}$. Temos $w \leq u_{n+1} \leq u_{n}$ e assim,

$$
\begin{aligned}
\varepsilon d\left(w, u_{n+1}\right) & \leq \phi\left(u_{n+1}\right)-\phi(w) \\
& \leq \inf _{S_{n}} \phi+\frac{1}{n+1}-\inf _{S_{n}} \phi \\
& =\frac{1}{n+1} .
\end{aligned}
$$

Deste modo,

$$
\begin{aligned}
\operatorname{diam} S_{n+1} & =\sup \left\{d\left(w_{1}, w_{2}\right) ; w_{1}, w_{2} \in S_{n+1}\right\} \\
& \leq d\left(w_{1}, u_{n+1}\right)+d\left(u_{n+1}, w_{2}\right) \\
& \leq \frac{1}{\varepsilon(n+1)}+\frac{1}{\varepsilon(n+1)} \\
& =\frac{2}{\varepsilon(n+1)}
\end{aligned}
$$

isto é, $\operatorname{diam} S_{n} \rightarrow 0$ quando $n \rightarrow \infty$. Como $X$ é completo,

$$
\bigcap_{n \in \mathbb{N}} S_{n}=\{v\}, \text { para algum } v \in X .
$$

Em particular, $v \in S_{0}$, isto é, $v \leq u_{0}=u$. Assim,

$$
\phi(v) \leq \phi(u)-\varepsilon d(v, u) \leq \phi(u),
$$

e, portanto,

$$
d(u, v) \leq \frac{\phi(u)-\phi(v)}{\varepsilon} \leq \frac{\inf \phi+\varepsilon-\inf \phi}{\varepsilon}=1 .
$$

Afirmamos que se $w \leq v$, então $w=v$. De fato, se $w \leq v$, então $w \leq u_{n}, \forall n \in \mathbb{N}$. Logo,

$$
w \in \bigcap_{n \in \mathbb{N}} S_{n} .
$$

Portanto, $w=v$.

Agora note que se $w \neq v$, então $w>v$, o que implica que $\phi(w)+\varepsilon d(v, w)>\phi(v)$, isto é:

$$
\phi(w)>\phi(v)-\varepsilon d(v, w),
$$

o que conclui a demonstração do teorema.

\subsubsection{A Condição de Palais-Smale}

Definição 1.2.1. Seja $X$ um espaço de Banach e $\phi: X \rightarrow \mathbb{R}$ um funcional de classe $\mathcal{C}^{1}$. Dizemos que $\phi$ satisfaz a condição de Palais-Smale no nível c (designada por $\left.(P S)_{c}\right)$ se toda sequência $\left(u_{n}\right)$ em $X$ que satisfaz

$$
\phi\left(u_{n}\right) \rightarrow c \quad e \quad \phi^{\prime}\left(u_{n}\right) \rightarrow 0,
$$

contém uma subsequência convergente em $X$. Se $\phi$ satisfizer a condição $(P S)_{c}$ para todo $c \in \mathbb{R}$, dizemos simplesmente que $\phi$ satisfaz a condição $(P S)$. 
Consideremos agora o seguinte problema:

$$
\left\{\begin{array}{l}
-\Delta v=a(x) v^{+}-b(x) v^{-}, \quad x \in \Omega \\
v=0, \quad x \in \partial \Omega .
\end{array}\right.
$$

onde, $a(x)$ e $b(x)$ são como em (2).

Teorema 1.2.2. Se a $(x)$ e b(x) satisfazem a condição (3) ou a condição (4), segue-se que o problema $\left(P^{ \pm}\right)$só possui a solução trivial.

\section{Demonstração:}

Demonstremos o teorema no caso da condição (4). O outro caso pode ser demonstrado analogamente. Defina

$$
\lambda=\frac{\lambda_{j}+\lambda_{j+1}}{2} \text { e } \quad \mu=\lambda_{j+1}-\lambda_{j}
$$

onde $\lambda_{j}$ são autovalores do operador Laplaciano com condição de Dirichlet (veja Apêndice D).

Escrevamos o problema $\left(P^{ \pm}\right)$na forma:

$$
\left\{\begin{array}{l}
-\Delta v-\lambda v=(a-\lambda) v^{+}-(b-\lambda) v^{-}, \quad x \in \Omega, \\
v=0, \quad x \in \partial \Omega
\end{array}\right.
$$

Seja $T: L^{2} \rightarrow L^{2}$ o operador linear assim definido: para cada $f \in L^{2}$, Tf é a única solução $w$ do problema

$$
\left\{\begin{array}{l}
-\Delta w-\lambda w=f, \quad x \in \Omega \\
w=0, \quad x \in \partial \Omega
\end{array}\right.
$$

Provemos que

$$
\|T\|=\frac{2}{\mu} .
$$

De fato, dado $f \in L^{2}(\Omega)$, seja $w \in L^{2}(\Omega)$ a solução de (1.6) que estamos buscando. Consideremos as sequências $\left\{a_{j}\right\}$ e $\left\{c_{j}\right\}$ em $\mathbb{R}$ tais que

$$
f=\sum_{j=1}^{\infty} c_{j} \varphi_{j} \quad \text { e } \quad w=\sum_{j=1}^{\infty} a_{j} \varphi_{j}
$$

onde $\varphi_{j}$ são as autofunções associadas aos $\lambda_{j}$ que compõe o espectro do operador $-\Delta$ com condição de Dirichlet. Com isto, o problema (1.6) se escreve como

$$
\sum_{j=1}^{\infty} a_{j}\left(\lambda_{j}-\lambda\right) \varphi_{j}=\sum_{j=1}^{\infty} c_{j} \varphi_{j}
$$

Logo,

$$
a_{j}=\frac{c_{j}}{\left(\lambda_{j}-\lambda\right)}
$$


A convergência da série de $w$ segue do fato que

$$
\left|a_{j}\right| \leq\left|c_{j}\right| \frac{1}{d}
$$

onde $d$ é a distância de $\lambda$ ao espectro, isto é, $d=\min \left\{\left|\lambda-\lambda_{j}\right| ; j=1,2, \cdots\right\}$. Da equação (1.7), segue que

$$
\|T\| \leq \frac{1}{d}
$$

Em (1.8) obtemos igualdade se resolvermos o problema

$$
\left\{\begin{array}{l}
-\Delta w-\lambda w=f, \quad x \in \Omega, \\
w=0, \quad x \in \partial \Omega,
\end{array}\right.
$$

onde $f=\varphi_{j}$ é a autofunção correspondente ao autovalor $\lambda_{j}$ mais próximo de $\lambda$. Mas, o problema acima possui uma solução pela Alternativa de Fredholm (Teorema B.0.5). Assim,

$$
\|T\|=\frac{1}{d}
$$

Notemos que $d=\frac{\mu}{2}$. Portanto,

$$
\|T\|=\frac{2}{\mu},
$$

como queríamos. Consequentemente o problema (1.5) é equivalente a

$$
v=T\left((a-\lambda) v^{+}-(b-\lambda) v^{-}\right) .
$$

Defina $S: L^{2}(\Omega) \rightarrow L^{2}(\Omega)$ por $S(v)=T\left((a-\lambda) v^{+}-(b-\lambda) v^{-}\right)$. Portanto, uma solução de (1.5) será um ponto fixo de $S$. Como $v=0$ é uma solução de (1.5), basta provar que $S$ é uma contração para concluir a demonstração do teorema. Seja $W: L^{2} \rightarrow L^{2}$, o operador definido por

$$
W(v)=(a-\lambda) v^{+}-(b-\lambda) v^{-} .
$$

Note que pela hipótese (4), temos:

$$
\|a(x)-\lambda\|_{L^{\infty}},\|b(x)-\lambda\|_{L^{\infty}} \leq \max \left\{\left|\lambda-c_{j}\right|,\left|\lambda-c_{j+1}\right|\right\}=: k
$$

e

$$
k<\frac{\mu}{2} .
$$

Seja $f(x, s)=(a(x)-\lambda) s^{+}-(b(x)-\lambda) s^{-}$. Para cada $x \in \Omega$ fixado, temos:

$$
\left|f\left(x, s_{1}\right)-f\left(x, s_{2}\right)\right| \leq k\left|s_{1}-s_{2}\right|, \forall s_{1}, s_{2} \in \mathbb{R} .
$$

Deste modo,

$$
\int_{\Omega}\left|W\left(v_{1}\right)(x)-W\left(v_{2}\right)(x)\right|^{2} d x \leq k^{2} \int_{\Omega}\left|v_{1}(x)-v_{2}(x)\right|^{2} d x .
$$

E, assim obtemos

$$
\left\|S v_{1}-S v_{2}\right\|_{L^{2}} \leq \frac{2 k}{\mu}\left\|v_{1}-v_{2}\right\|_{L^{2}},
$$

o que mostra que $S$ é uma contração. 
Teorema 1.2.3. Suponha que $f \in \mathcal{C}(\bar{\Omega} \times \mathbb{R}, \mathbb{R})$. Suponha também que o problema ( $\left.P^{ \pm}\right)$só possui a solução trivial $v=0$. Então o funcional $\phi$ satisfaz a condição de Palais-Smale.

\section{Demonstração:}

Seja $\left\{u_{n}\right\}$ uma subsequência em $H_{0}^{1}(\Omega)$ tal que

$$
\left|\phi\left(u_{n}\right)\right|=\left.\left|\frac{1}{2} \int_{\Omega}\right| \nabla u_{n}\right|^{2} d x-\int_{\Omega} F\left(x, u_{n}\right) d x \mid \leq C,
$$

para alguma constante $C>0 \mathrm{e}$

$$
\left|\phi^{\prime}\left(u_{n}\right) v\right|=\left|\int_{\Omega} \nabla u_{n} \nabla v d x-\int_{\Omega} f\left(x, u_{n}\right) v d x\right| \leq \varepsilon_{n}\|v\|_{H_{0}^{1}}, \forall v \in H_{0}^{1}(\Omega)
$$

$\operatorname{com} \varepsilon_{n} \rightarrow 0$ quando $n \rightarrow \infty$. Afirmamos que $\left\{u_{n}\right\}$ é uma sequência limitada em $H_{0}^{1}(\Omega)$. De fato, suponha por contradição que

$$
\left\|u_{n}\right\|_{H_{0}^{1}(\Omega)} \rightarrow \infty, \quad \text { quando } n \rightarrow 0
$$

Seja $v_{n} \doteq \frac{u_{n}}{\left\|u_{n}\right\|_{H_{0}^{1}}}$. Note que existem $v_{0} \in H_{0}^{1}(\Omega)$ e uma subsequência, ainda denotada por $\left\{v_{n}\right\}_{n}$, tais que

$$
v_{n} \rightarrow v_{0} \text { fracamente em } H_{0}^{1}(\Omega) \quad \text { e } \quad v_{n} \rightarrow v_{0} \text { fortemente em } L^{2}(\Omega) .
$$

Afirmação 1: A subsequência $\left\{v_{n}\right\}_{n}$ acima pode ser tomada de modo que

$$
v_{n}(x) \rightarrow v_{0}(x) \text { q.s. em } \Omega \quad \text { e }\left|v_{n}(x)\right| \leq h(x),
$$

para alguma $h \in L^{2}(\Omega)$.

De fato, como $v_{n} \rightarrow v_{0}$ na norma de $L^{2}(\Omega)$, segue que a sequência $\left\{v_{n}\right\}_{n}$ é de Cauchy em $L^{2}(\Omega)$. Assim, podemos tomar subsequência $\left\{v_{n_{k}}\right\}$ com

$$
\left\|v_{n_{k+1}}-v_{n_{k}}\right\|_{L^{2}(\Omega)} \leq \frac{1}{2^{k}}, \forall k \geq 1
$$

Para facilitar a notação denotemos por $v_{k}$ o elemento $v_{n_{k}}$. Definamos, $\forall n \in \mathbb{N}$,

$$
g_{n}(x)=\sum_{k=1}^{n}\left|v_{k+1}(x)-v_{k}(x)\right| \text {. }
$$

Desse modo,

$$
\left\|g_{n}\right\|_{L^{2}(\Omega)} \leq 1
$$

Pelo Teorema da Convergência Monótona, $g_{n}(x)$ converge para um limite que denotaremos por $g(x)$, onde $g \in L^{2}(\Omega)$. Tomemos $m \geq n \geq 2$. Temos:

$$
\left|v_{m}(x)-v_{n}(x)\right| \leq\left|v_{m}(x)-v_{m-1}(x)\right|+\cdots+\left|v_{n+1}(x)-v_{n}(x)\right| \leq g(x)-g_{n-1}(x) .
$$


Como para quase todo $x \in \Omega,\left\{v_{n}(x)\right\}_{n}$ é uma sequência de Cauchy em $\mathbb{R}$, existe $v$ tal que $v_{n}(x) \rightarrow v(x)$ pontualmente. Assim,

$$
\left|v(x)-v_{n}(x)\right| \leq g(x), \quad \text { para } n \geq 2 .
$$

Pela equação acima, $v \in L^{2}(\Omega)$, e pelo Teorema da Convergência Dominada de Lebesgue,

$$
v_{n_{k}} \rightarrow v \quad \text { em } L^{2}(\Omega)
$$

Portanto, $v=v_{0}$. Para obter o segundo fato, basta tomarmos $h=v+g$.

Mostremos agora que

$$
\frac{f\left(x, u_{n}(x)\right)}{\left\|u_{n}\right\|_{H_{0}^{1}(\Omega)}} \rightarrow a(x) v_{0}^{+}-b(x) v_{0}^{-} \text {fracamente em } L^{2}(\Omega) .
$$

De fato, defina

$$
\alpha_{n}=\left\|u_{n}\right\|_{H_{0}^{1}(\Omega)} \quad \text { e } \quad l(x)=a(x) v_{0}^{+}-b(x) v_{0}^{-} .
$$

É suficiente provar que toda subsequência de

$$
f_{n}(x)=\frac{f\left(x, \alpha_{n} v_{n}(x)\right)}{\alpha_{n}}
$$

possui subsequência convergente para $l(x)$ em $L^{2}(\Omega)$. Pela equação (1.1), temos

$$
\left|f_{n}(x)\right|=\frac{1}{\alpha_{n}}\left|f\left(x, \alpha_{n} v_{n}(x)\right)\right| \leq c_{1}\left|v_{n}(x)\right|+\frac{c_{2}}{\alpha_{n}} \leq c_{1} h(x)+\frac{c_{2}}{\alpha_{n}} .
$$

Como $h \in L^{2}(\Omega)$ e $\alpha_{n} \rightarrow \infty$ quando $n \rightarrow \infty,\left\{f_{n}\right\}$ é uma sequência limitada em $L^{2}(\Omega)$. Portanto, existe uma subsequência, ainda denotada por $\left\{f_{n}\right\}$, fracamente convergente em $L^{2}(\Omega)$.

Por outro lado, consideremos o conjunto

$$
A=\left\{x ; v_{0}(x) \neq 0\right\} .
$$

Note que em $A^{+}=\left\{x \in A ; v_{0}(x)>0\right\}$,

$$
\frac{f\left(x, \alpha_{n} v_{n}\right)}{\alpha_{n} v_{n}} v_{n} \rightarrow a(x) v_{0}^{+}, \quad \text { para quase todo } x \in A^{+},
$$

e em $A^{-}=\left\{x \in A ; v_{0}(x)<0\right\}$,

$$
\frac{f\left(x, \alpha_{n} v_{n}\right)}{\alpha_{n} v_{n}} v_{n} \rightarrow-b(x) v_{0}^{-}, \quad \text { para quase todo } x \in A^{-} .
$$

Portanto,

$$
f_{n}(x) \rightarrow l(x), \quad \text { para quase todo } x \in A .
$$

Seja agora $B=\left\{x ; v_{0}(x)=0\right\}$. Por (1.13) e usando que $\alpha_{n} \rightarrow 0$, temos

$$
f_{n}(x) \rightarrow 0, \quad \text { para quase todo } x \in B
$$


Portanto,

$$
f_{n}(x) \rightarrow a(x) v_{0}^{+}-b(x) v_{0}^{-}, \quad \text { para quase todo } x \in \Omega, \text { quando } n \rightarrow \infty .
$$

Pela unicidade do limite, $f_{n} \rightarrow a v_{0}^{+}-b v_{0}^{-}$fracamente em $L^{2}(\Omega)$. Agora, dividindo a equação (1.10) por $\alpha_{n}$ e tomando o limite com $n \rightarrow \infty$, obtemos:

$$
\int_{\Omega} \nabla v_{0} \nabla v d x-\int_{\Omega}\left[a(x) v_{0}^{+}-b(x) v_{0}^{-}\right] v d x=0, \forall v \in H_{0}^{1}(\Omega) .
$$

Como $v_{0}$ é solução de $\left(P^{ \pm}\right)$, segue do Teorema 1.2.2 que $v_{0}=0$. Fazendo $v=v_{n} /\left\|u_{n}\right\|_{H_{0}^{1}(\Omega)}^{2}$ em (1.10), obtemos

$$
\left.\left|\int_{\Omega}\right| \nabla v_{n}\right|^{2} d x-\int_{\Omega} \frac{f\left(x, u_{n}\right)}{\alpha_{n}} v_{n} d x \mid \leq \frac{\varepsilon_{n}}{\alpha_{n}}
$$

e, assim,

$$
\left|1-\int_{\Omega} \frac{f\left(x, u_{n}\right)}{\alpha_{n}} v_{n} d x\right| \leq \frac{\varepsilon_{n}}{\alpha_{n}} .
$$

Por outro lado,

$$
\int_{\Omega} \frac{f\left(x, u_{n}\right)}{\alpha_{n}} v_{n} d x \rightarrow \int_{\Omega}\left[a(x) v_{0}^{+}-b(x) v_{0}^{-}\right] v_{0} d x=0 \quad \text { e } \quad \frac{\varepsilon_{n}}{\alpha_{n}} \rightarrow 0,
$$

o que leva a um absurdo ao fazer $n \rightarrow \infty$ em (1.14). Portanto, a sequência $\left\{u_{n}\right\}$ é limitada em $H_{0}^{1}(\Omega)$, o que verifica a afirmação.

Em consequência da limitação de $\left\{u_{n}\right\}$ em $H_{0}^{1}(\Omega)$, existe uma subsequência $\left\{u_{n_{j}}\right\}$ que converge fracamente para $u_{0}$ em $H_{0}^{1}(\Omega)$ e converge na norma para o mesmo $u_{0}$ em $L^{p}(\Omega)$, com $1 \leq p<2^{*}$. Por outro lado, a equação de (1.10) nos diz que

$$
\left|\int_{\Omega} \nabla u_{n} \nabla v d x-\int_{\Omega} f\left(x, u_{n}\right) v d x\right| \leq \varepsilon_{n}\|v\|_{H_{0}^{1}}, \forall v \in H_{0}^{1}(\Omega)
$$

onde, $\varepsilon_{n} \rightarrow 0$. Seja $v=u_{n}-u_{0}$. Por (1.1), existe $C>0$ tal que

$$
|f(x, s)| \leq C(1+|s|), \quad \forall x \in \bar{\Omega}, s \in \mathbb{R} .
$$

Pela desigualdade de Hölder,

$$
\begin{aligned}
\left|\int_{\Omega} f\left(x, u_{n_{j}}\right)\left(u_{n_{j}}-u_{0}\right) d x\right| & \leq\left(\int_{\Omega}\left|f\left(x, u_{n_{j}}\right)\right|^{2} d x\right)^{\frac{1}{2}}\left(\int_{\Omega}\left|u_{n_{j}}-u_{0}\right|^{2} d x\right)^{\frac{1}{2}} \\
& \leq C\left[\int_{\Omega}\left(1+\left|u_{n_{j}}\right|\right)^{2} d x\right]^{\frac{1}{2}}\left\|u_{n_{j}}-u_{0}\right\|_{L^{2}(\Omega)} \rightarrow 0
\end{aligned}
$$

uma vez que $\left\|u_{n_{j}}-u_{0}\right\|_{L^{2}(\Omega)} \rightarrow 0$ e $\left[\int_{\Omega}\left(1+\left|u_{n_{j}}\right|\right)^{2}\right]^{\frac{1}{2}}$ é limitado. Assim,

$$
\int_{\Omega}\left(\nabla u_{n_{j}} \nabla\left(u_{n_{j}}-u_{0}\right)\right) d x \rightarrow 0
$$

e usando que $\left\{u_{n_{j}}\right\}$ converge fracamente para $u_{0}$ em $H_{0}^{1}(\Omega)$, obtemos

$$
\int_{\Omega}\left|\nabla u_{n_{j}}\right|^{2} d x \rightarrow \int_{\Omega}\left|\nabla u_{0}\right|^{2} d x .
$$

Portanto, $\left\{u_{n_{j}}\right\}$ converge fortemente para $u_{0}$ em $H_{0}^{1}(\Omega)$, como queríamos. 


\subsubsection{Demonstração do Teorema 0.0.1}

Sabemos que o funcional $\phi: H_{0}^{1}(\Omega) \rightarrow \mathbb{R}$ é contínuo e limitado inferiormente. Logo, estamos em condição de aplicar o princípio variacional de Ekeland. Assim, dado $\varepsilon=\frac{1}{n}$, existe $u_{n} \in H_{0}^{1}(\Omega)$ tal que

$$
\phi\left(u_{n}\right) \leq \inf _{H_{0}^{1}(\Omega)} \phi+\frac{1}{n}
$$

e

$$
\phi\left(u_{n}\right) \leq \phi(u)+\frac{1}{n} d\left(u, u_{n}\right), \forall u \in H_{0}^{1}(\Omega) .
$$

$\operatorname{Em}(1.16)$, tomemos $u=u_{n}+t w$, onde $t>0$ e $\|w\|_{H_{0}^{1}(\Omega)}=1$. Assim, obtemos:

$$
\phi\left(u_{n}\right) \leq \phi\left(u_{n}+t w\right)+\frac{1}{n} t
$$

isto é,

$$
\frac{\phi\left(u_{n}\right)-\phi\left(u_{n}+t w\right)}{t} \leq \frac{1}{n} .
$$

Tomando o limite quando $t \rightarrow 0$,

$$
-\phi^{\prime}\left(u_{n}\right) w \leq \frac{1}{n}, \forall w \in H_{0}^{1}(\Omega), \operatorname{com}\|w\|_{H_{0}^{1}(\Omega)}=1 .
$$

Deste modo,

$$
\left|\phi^{\prime}\left(u_{n}\right) w\right| \leq \frac{1}{n}, \forall w \in H_{0}^{1}(\Omega), \operatorname{com}\|w\|_{H_{0}^{1}(\Omega)}=1
$$

Logo,

$$
\left\|\phi^{\prime}\left(u_{n}\right)\right\| \leq \frac{1}{n} .
$$

Note agora que, quando $n \rightarrow \infty$, por (1.15),

$$
\phi\left(u_{n}\right) \rightarrow c=\inf _{H_{0}^{1}(\Omega)} \phi,
$$

e, por (1.17),

$$
\phi^{\prime}\left(u_{n}\right) \rightarrow 0 \text {. }
$$

Pelo Teorema 1.2.3 sabemos que $\phi$ satisfaz (PS). Assim, existem uma subsequência $\left\{u_{n_{j}}\right\}$ de $\left\{u_{n}\right\}$ e $u_{0} \in H_{0}^{1}(\Omega)$ tais que $u_{n_{j}} \rightarrow u_{0}$. Como $\phi$ é um funcional de classe $\mathcal{C}^{1}$ (ver Apêndice $\mathrm{C})$, temos que $\phi\left(u_{n_{j}}\right) \rightarrow \phi\left(u_{0}\right)$, e por (1.15), fazendo $n \rightarrow \infty$ temos

$$
\phi\left(u_{0}\right) \leq \inf _{H_{0}^{1}(\Omega)} \phi .
$$

Mas, $\inf \left\{\phi(u) ; u \in H_{0}^{1}(\Omega)\right\} \leq \phi\left(u_{0}\right)$, portanto,

$$
\phi\left(u_{0}\right)=\inf _{H_{0}^{1}(\Omega)} \phi .
$$

Novamente, como $\phi$ é um funcional de classe $\mathcal{C}^{1}$,

$$
u_{n_{j}} \rightarrow u_{0} \Rightarrow \phi^{\prime}\left(u_{n_{j}}\right) \rightarrow \phi^{\prime}\left(u_{0}\right) \Rightarrow\left\|\phi^{\prime}\left(u_{n_{j}}\right)\right\| \rightarrow\left\|\phi^{\prime}\left(u_{0}\right)\right\| .
$$

Além disso, por (1.17) temos que $\left\|\phi^{\prime}\left(u_{n_{j}}\right)\right\| \rightarrow 0$, e portanto, $\left\|\phi^{\prime}\left(u_{0}\right)\right\|=0$, o que implica que

$$
\phi^{\prime}\left(u_{0}\right)=0,
$$

mostrando que $u_{0}$ é um ponto crítico de $\phi$, e portanto, uma solução fraca do problema (1). 


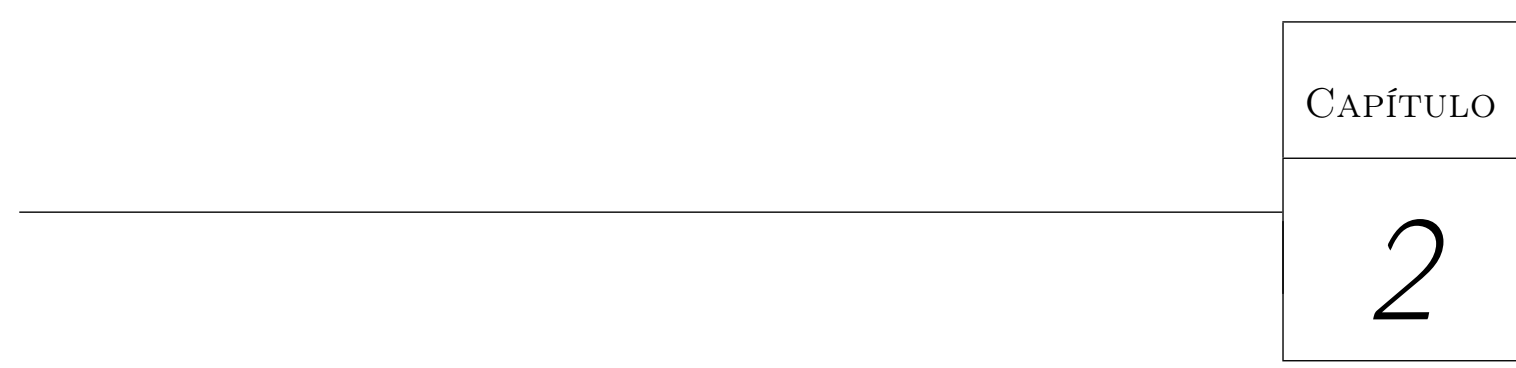

Teorema 0.0 .2

Este capítulo é dedicado à prova do Teorema 0.0.2. A primeira seção é dedicada em apresentar uma demonstração empregando a teoria do grau topológico. Na segunda seção empregaremos o teorema do ponto de sela para dar uma segunda demonstração.

\subsection{Demonstração via teoria do grau}

O primeiro resultado a seguir é crucial para a utilização da teoria do grau.

\subsubsection{Estimativa a Priori}

Teorema 2.1.1. Suponha que o problema (1) é assintoticamente linear com $a(x), b(x) \in$ $L^{\infty}(\Omega)$. Suponha também que a única solução $v \in H_{0}^{1}(\Omega)$ de

$$
\left\{\begin{array}{l}
-\Delta v=a(x) v^{+}-b(x) v^{-}, \quad x \in \Omega, \\
v=0, \quad x \in \partial \Omega,
\end{array}\right.
$$

é a solução trivial. Então, existe $K>0$ tal que

$$
\|u\|_{H_{0}^{1}(\Omega)} \leq K
$$

para todas as eventuais soluções de (1).

Observação 2.1.1. Note que pelo Teorema (1.2.2), se $a(x)$ e $b(x)$ satisfazem as hipóteses (3) ou (4), então o problema $\left(P^{ \pm}\right)$só possui a solução trivial. 


\section{Demonstração do Teorema 2.1.1:}

Suponha, por absurdo, que existe uma sequência $\left\{u_{n}\right\} \in H_{0}^{1}(\Omega)$ de soluções de (1) tal que $\left\|u_{n}\right\|_{H_{0}^{1}(\Omega)} \rightarrow \infty$. Defina $v_{n}=u_{n} /\left\|u_{n}\right\|_{H_{0}^{1}(\Omega)}$. Como visto na demonstração do Teorema 0.0.1 via método variacional, existe uma subsequência de $\left\{v_{n}\right\}$ também denotada por $\left\{v_{n}\right\}$, e um $v_{0} \in H_{0}^{1}(\Omega)$ tais que

$$
v_{n} \rightarrow v_{0} \quad \text { fracamente em } H_{0}^{1}(\Omega)
$$

e

$$
v_{n} \rightarrow v_{0} \quad \text { fortemente } L^{2}(\Omega) .
$$

Como visto no capítulo anterior, esta subsequência pode ser tomada de modo que

$$
v_{n}(x) \rightarrow v_{0}(x) \quad \text { quase sempre em } \Omega
$$

$\mathrm{e}$

$$
\left|v_{n}(x)\right| \leq h(x)
$$

para alguma $h \in L^{2}(\Omega)$. Agora, como $u_{n}$ é solução fraca de (1), temos:

$$
\int_{\Omega} \nabla v_{n} \cdot \nabla \varphi d x=\int_{\Omega} \frac{f\left(x, u_{n}\right)}{\left\|u_{n}\right\|_{H_{0}^{1}(\Omega)}} \varphi d x, \forall \varphi \in H_{0}^{1}(\Omega) .
$$

Repetindo os argumentos da verificação da Afirmação 1 da prova do Teorema 1.2.3, temos

$$
\frac{f\left(x, u_{n}\right)}{\left\|u_{n}\right\|_{H_{0}^{1}(\Omega)}} \rightarrow a(x) v_{0}^{+}-b(x) v_{0}^{-} \quad \text { fracamente em } L^{2}(\Omega) .
$$

Tomando o limite em (2.1), obtemos:

$$
\int_{\Omega} \nabla v_{0} \cdot \nabla \varphi d x=\int_{\Omega}\left(a(x) v_{0}^{+}-b(x) v_{0}^{-}\right) \varphi d x
$$

de onde segue que $v_{0}=0$, em vista da hipótese. Por outro lado tomando $\varphi=v_{n}$ em (2.1), obtemos

$$
1=\int_{\Omega}\left|\nabla v_{n}\right|^{2} d x=\int_{\Omega} \frac{f\left(x \cdot u_{n}\right)}{\left\|u_{n}\right\|_{H_{0}^{1}(\Omega)}} v_{n} d x .
$$

Por (2.2) e o fato que $v_{n} \rightarrow v_{0}=0$ em $L^{2}(\Omega)$, obtemos um absurdo ao fazer o limite na equação acima. Portanto, existe $K>0$, tal que

$$
\|u\|_{H_{0}^{1}(\Omega)} \leq K
$$

para qualquer que seja $u$ uma solução do problema (1).

\subsubsection{Demonstração do Teorema 0.0.2}

Defina

$$
g(x, u)=\theta \lambda u+(1-\theta) f(x, u), \quad \operatorname{com} \theta \in[0,1] \quad \text { e } \lambda=\frac{\lambda_{j}+\lambda_{j+1}}{2} .
$$


Assim, temos:

$$
\lim _{s \rightarrow \infty} \frac{g(x, s)}{s}=\lim _{s \rightarrow \infty}\left[\frac{\theta \lambda s}{s}+(1-\theta) \frac{f(x, s)}{s}\right]=\theta \lambda+(1-\theta) a(x)=a_{\theta}(x)
$$

e

$$
\lim _{s \rightarrow-\infty} \frac{g(x, s)}{s}=\theta \lambda+(1-\theta) b(x)=b_{\theta}(x) .
$$

Note que $a_{\theta}(x), b_{\theta}(x) \in L^{\infty}(\Omega)$ para todo $\theta \in[0,1]$, pois $a(x), b(x) \in L^{\infty}(\Omega)$. Pela condição $(4)$

$$
\lambda_{j}<c_{j} \leq a(x), b(x) \leq c_{j+1}<\lambda_{j+1},
$$

temos que

$$
\lambda_{j}<c_{j} \leq a_{\theta}(x), b_{\theta}(x) \leq c_{j+1}<\lambda_{j+1}, \forall \theta \in[0,1] .
$$

Consideremos agora a família de problemas

$$
\left\{\begin{array}{l}
-\Delta u=\theta \lambda u+(1-\theta) f(x, u), \quad x \in \Omega, \\
u=0, \quad x \in \partial \Omega .
\end{array}\right.
$$

Como $a_{\theta}(x)$ e $b_{\theta}(x)$ satisfazem a condição (4), pelo Teorema 1.2.2, o problema $\left(P^{ \pm}\right)$, com $a(x)=a_{\theta}(x)$ e $b(x)=b_{\theta}(x)$, só possui a solução trivial. Deste modo, pelo Teorema 2.1.1, existem $K(\theta)>0$ tal que $\|u\|_{H_{0}^{1}(\Omega)} \leq K(\theta)$, para todas as eventuais soluções de $\left(P_{\theta}\right)$.

Mostremos que $\mathrm{K}$ independe de $\theta$. Para tanto, basta mostrar que $\{K(\theta) ; \theta \in[0,1]\}$ é limitado. De fato, suponha por contradição que exista uma sequência $\left\{\theta_{n}\right\} \subset[0,1]$ e uma família de soluções $\left\{u_{\theta_{n}}\right\}$, com $\left\|u_{\theta_{n}}\right\|_{H_{0}^{1}(\Omega)} \rightarrow \infty$ quando $n \rightarrow \infty$. Tomando $v_{n}=u_{\theta_{n}} /\left\|u_{\theta_{n}}\right\|_{H_{0}^{1}(\Omega)}$ e repetindo os argumentos da demonstração do Teorema 2.1.1 obtemos a mesma contradição.

Designemos por $S: L^{2} \rightarrow L^{2}$ o inverso do operador $-\Delta$, sujeito a condição de Dirichlet, e por $T_{\theta}: L^{2} \rightarrow L^{2}$, o operador definido por

$$
T_{\theta}(u)=S(\theta \lambda u+(1-\theta) f(\cdot, u)) .
$$

Como S é um operador compacto, então $T_{\theta}$ também o será, pois trata-se da composição de um operador compacto com um contínuo. Deste modo, $I-T_{\theta}$ será uma perturbação compacta da identidade.

Notemos também que $\left(P_{\theta}\right)$ é equivalente a $\left(I-T_{\theta}\right) u=0$. Assim, $\left(I-T_{\theta}\right) u \neq 0$, para $u \in H_{0}^{1}(\Omega) \operatorname{com}\|u\|_{H_{0}^{1}(\Omega)}=K+1$. Além disso, pelo Teorema E.2.1,

$$
d\left(I-T_{1}, B_{r}(0), 0\right)= \pm 1 \neq 0, \forall r>0 .
$$

Logo,

$$
d\left(I-T_{1}, B_{K+1}(0), 0\right) \neq 0
$$

e como o grau é invariante por homotopia, considerando a homotopia $H(u, \theta)=I-T_{\theta}$, temos:

$$
d\left(I-T_{0}, B_{K+1}(0), 0\right) \neq 0 .
$$

Pela Propriedade E.2.2(3), existe $u \in B_{K+1}(0)$ tal que $\left(I-T_{0}\right) u=0$, implicando que $u$ é solução do problema (1), como queríamos. 


\subsection{Demonstração via o teorema do ponto de sela}

Neste capítulo nossa ferramenta para a demonstração do Teorema 0.0.2 será o teorema do ponto de sela [11], e para o estudo de tal teorema será necessário estudarmos previamente o Lema de Deformação $[1,4,10]$.

\subsubsection{Lema de Deformação}

Definição 2.2.1. Seja $\phi: X \rightarrow \mathbb{R}$ um funcional de classe $\mathcal{C}^{1}$ num espaço de Banach $X$. Dizemos que $c \in \mathbb{R}$ é um valor crítico de $\phi$ se $\phi(u)=c$ para algum ponto crítico $u \in X$ de $\phi$.

O conjunto de todos os pontos críticos no nível $c$ será denotado por $K_{c}$, isto é,

$$
K_{c}=\left\{u \in X ; \phi^{\prime}(u)=0 \text { e } \phi(u)=c\right\}
$$

Também designaremos por $\phi^{c}$ o conjunto de todos os pontos em nível menor ou igual a $c$, isto é,

$$
\phi^{c}=\{u \in X ; \phi(u) \leq c\} .
$$

Dado $S \subset X$, denotemos por $S_{\alpha}$ a vizinhança fechada de $S$ definida por

$$
S_{\alpha}=\{u \in X ; \operatorname{dist}(u, S) \leq \alpha\}
$$

Definição 2.2.2. [10] Um campo pseudo-gradiente para $\phi \in \mathcal{C}^{1}(X, \mathbb{R})$ é uma aplicação localmente Lipschitziana $v: Y \rightarrow X$, onde $Y=\left\{u \in X ; \phi^{\prime}(u) \neq 0\right\}$, satisfazendo:

(i) $\|v(u)\| \leq 2\left\|\phi^{\prime}(u)\right\|, \forall u \in Y$;

(ii) $\phi^{\prime}(u) \cdot v(u) \geq\left\|\phi^{\prime}(u)\right\|^{2}, \forall u \in Y$.

Lema 2.2.1. [1, 4] Seja $\phi: X \rightarrow \mathbb{R}$ um funcional de classe $\mathcal{C}^{1}$ no espaço de Banach $X$. Suponha que $S \subset X, c \in \mathbb{R} e \varepsilon, \delta>0$ são tais que

$$
\left\|\phi^{\prime}(u)\right\| \geq \frac{4 \varepsilon}{\delta}, \forall u \in \phi^{-1}([c-2 \varepsilon, c+2 \varepsilon]) \cap S_{2 \delta} .
$$

Então, existe $\eta \in \mathcal{C}([0,1] \times X, X)$ tal que para todos $u \in X$ e $t \in[0,1]$, tem-se

(i) $\eta(0, u)=u$;

(ii) $\eta(t, u)=u$, se $u \notin \phi^{-1}([c-2 \varepsilon, c+2 \varepsilon]) \cap S_{2 \delta}$;

(iii) $\eta\left(1, \phi^{c+\varepsilon} \cap S\right) \subset \phi^{c-\varepsilon} \cap S_{\delta}$;

(iv) $\eta(t, \cdot): X \rightarrow X$ é um homeomorfismo. 


\section{Demonstração:}

Sejam

$$
\begin{gathered}
A=\phi^{-1}([c-2 \varepsilon, c+2 \varepsilon]) \cap S_{2 \delta}, \\
B=\phi^{-1}([c-\varepsilon, c+\varepsilon]) \cap S_{\delta} \quad \mathrm{e} \\
Y=\left\{u \in X ; \phi^{\prime}(u) \neq 0\right\} .
\end{gathered}
$$

Note que $B \subset A \subset Y$. Seja $v: Y \rightarrow X$ um campo pseudo-gradiente para $\phi$, cuja existência é garantida pelo Lema A.2 [7], e considere uma função localmente Lipschitziana $\rho: X \rightarrow \mathbb{R}$ tal que $0 \leq \rho \leq 1 \mathrm{e}$

$$
\begin{gathered}
\rho \equiv 1 \quad \text { em } B \\
\rho \equiv 0 \quad \text { em } X \backslash A .
\end{gathered}
$$

Definamos então a seguinte aplicação localmente Lipschitziana $f: X \rightarrow X$

$$
f(u)=-\rho(u) \frac{v(u)}{\|v(u)\|} .
$$

Assim, $\|f(u)\| \leq 1$ para todo $u \in X$. Portanto, pelo teorema de existência e unicidade de equações diferenciais ordinárias, o problema de Cauchy

$$
\left\{\begin{array}{l}
\dot{w}=f(w) \\
w(0)=u
\end{array}\right.
$$

tem, para cada $u \in X$ dado, uma única solução definida para todo $t \geq 0$. Definamos $\eta:[0,1] \times X \rightarrow X$ dada por $\eta(t, u)=w(\delta t, u)$. Note que

$$
\eta(0, u)=w(0, u)=u,
$$

o que mostra $(i)$. Seja agora $u \notin \phi^{-1}([c-2 \varepsilon, c+2 \varepsilon]) \cap S_{2 \delta}$, ou seja, $u \notin A$. Logo, $\rho(u)=0$, e consequentemente $f(u)=0$. Assim, $(i i)$ está satisfeita.

Para mostrar (iii), note que, para $t \geq 0$,

$$
\|w(t, u)-u\| \leq \int_{0}^{t}\|f(w(\tau, u))\| d \tau \leq t
$$

Logo, $w(t, S) \subset S_{\delta}$, para todo $t \in[0, \delta]$. Pela definição de $\eta$, segue que

$$
\eta(t, S) \subset S_{\delta}, \forall t \in[0,1] .
$$

Note também que, para cada $u \in X$ fixado, a função $t \mapsto \phi(w(t, u))$ é não-crescente, pois:

$$
\begin{aligned}
\frac{d}{d t} \phi(w(t, u)) & =\phi^{\prime}(w(t, u)) \cdot w^{\prime}(t, u) \\
& =\phi^{\prime}(w(t, u)) \cdot f(w(t, u)) \\
& =-\rho(w(t, u)) \cdot \frac{v(w(t, u))}{\|v(w(t, u))\|} \cdot \phi^{\prime}(w(t, u)) \\
& \leq-\rho(w(t, u)) \cdot \frac{\left\|\phi^{\prime}(w(t, u))\right\|^{2}}{\|v(w(t, u))\|} \leq 0 .
\end{aligned}
$$

Seja agora $u \in \phi^{c+\varepsilon} \cap S$. Temos dois casos a serem considerados: 
(a) Se $\phi(w(\hat{t}, u))<c-\varepsilon$ para algum $\hat{t} \in[0, \delta)$, então

$$
\phi(\eta(1, u))=\phi(w(\delta, u)) \leq \phi(w(\hat{t}, u))<c-\varepsilon
$$

e, portanto, $\eta(1, u) \in \phi^{c-\varepsilon} \cap S_{\delta}$, em vista de (2.5).

(b) No outro caso, temos $w(t, u) \in \phi^{-1}([c-\varepsilon, c+\varepsilon]) \cap S_{\delta}=B$, para todo $t \in[0, \delta]$. Pelas equações (2.6) e (2.4), e usando que $\rho \equiv 1 \mathrm{em} B$ e a Definição 2.2.2, obtemos a seguinte desigualdade:

$$
\begin{aligned}
\phi(w(\delta, u)) & =\phi(u)+\int_{0}^{\delta} \frac{d}{d t} \phi(w(t, u)) d t \\
& \leq \phi(u)-\int_{0}^{\delta} \frac{1}{2}\left\|\phi^{\prime}(w(t, u))\right\| d t \\
& \leq c+\varepsilon-\frac{1}{2} \frac{4 \varepsilon}{\delta} \delta \\
& =c-\varepsilon .
\end{aligned}
$$

Portanto, tanto em (a) quanto em $(b), \eta(1, u)=w(\delta, u) \in \phi^{c-\varepsilon} \cap S_{\delta}$, quando $u \in \phi^{c+\varepsilon} \cap S$, o que verifica (iii). A prova do item $(i v)$ é feita utilizando propriedades de semigrupo para a solução do problema de Cauchy acima.

Lema 2.2.2. [4] (Lema de Deformação - Versão de Clark) Suponha que $\phi \in \mathcal{C}^{1}(X, \mathbb{R})$ satisfaz a condição $(P S)$. Se $c \in \mathbb{R}$ não é um valor crítico de $\phi$, então para todo $\varepsilon>0$ suficientemente pequeno, existe $\eta \in \mathcal{C}([0,1] \times X, X)$ tal que para todo $u \in X$ e $t \in[0,1]$, temos:

(i) $\eta(0, u)=u$;

(ii) $\eta(t, u)=u$ se $u \notin \phi^{-1}([c-2 \varepsilon, c+2 \varepsilon])$;

(iii) $\eta\left(1, \phi^{c+\varepsilon}\right) \subset \phi^{c-\varepsilon}$;

(iv) $\eta(t, \cdot): X \rightarrow X$ é um homeomorfismo.

\section{Demonstração:}

Devem existir constantes $\alpha, \beta>0$ tais que:

$$
u \in \phi^{-1}([c-2 \alpha, c+2 \alpha]) \Rightarrow\left\|\phi^{\prime}(u)\right\| \geq \beta .
$$

De fato, pois caso contrário, existiria uma sequência $\left\{u_{n}\right\}_{n}$ com

$$
c-\frac{1}{n} \leq \phi\left(u_{n}\right) \leq c+\frac{1}{n} \quad \text { e } \quad\left\|\phi^{\prime}(u)\right\| \leq \frac{1}{n} .
$$

E, pela condição $(P S)$, $c$ seria um valor crítico de $\phi$, o que é um absurdo. O resultado segue do Lema de deformação ao tomar $S=X, \varepsilon \in(0, \alpha]$ fixado e $\delta=\frac{4 \varepsilon}{\beta}$.

O resultado a seguir é crucial para estabelecer o teorema do ponto de sela e sua demonstração utiliza o grau topológico de Brouwer. 
Lema 2.2.3. Sejam $X$ um espaço de Banach, $V$ um subespaço de $X$ com dimensão finita $e$ $W$ seu complemento topológico, isto é, $X=V \oplus W$. Considere

$$
\Gamma=\left\{\gamma: \overline{B_{R}(0)} \cap V \rightarrow X ; \gamma \text { é contínua, } \gamma(u)=u, \forall u \in \partial B_{R}(0) \cap V\right\}
$$

Então dada $\gamma \in \Gamma$, existe $u_{0} \in B_{R}(0) \cap V$ tal que $\gamma\left(u_{0}\right) \in W$.

\section{Demonstração:}

Seja $P: X \rightarrow V$ a projeção de $X$ sobre $V$ associada à decomposição $X=V \oplus W$. Tal operador é linear, contínuo e é definido do seguinte modo: dado $u \in X, u$ se escreve de modo único como $u=v+w$, com $v \in V$ e $w \in W$. Assim, $P u=v$.

Definamos a seguinte aplicação:

$$
\begin{array}{cl}
S: \overline{B_{R}(0)} \cap V & \rightarrow V \\
v & \mapsto S(v)=P(\gamma(v)) .
\end{array}
$$

Considere agora a homotopia:

$$
H(\cdot, t)=(1-t) I+t S, \quad 0 \leq t \leq 1 .
$$

Observe que para $v \in \partial B_{R}(0) \cap V$, temos $S v=v$. Assim, $H(v, t)=v \neq 0$, para todo $t \in[0,1]$ e $v \in \partial B_{R}(0) \cap V$. Pela propriedade (2), Apêndice E.1.4, $d\left(H(\cdot, t), B_{R}(0) \cap V, 0\right)$ é constante em $[0,1]$. Sendo $H(\cdot, 0)=I$, tal constante deverá ser igual a 1 . Sendo $H(\cdot, 1)=S$, pela propriedade (3) do Apêndice E.1.5, existe $u_{0} \in B_{R}(0) \cap V$ tal que $S\left(u_{0}\right)=0$. Mas, isto que dizer que $P\left(\gamma\left(u_{0}\right)\right)=0$. Portanto, $\gamma\left(u_{0}\right) \in W$, como queríamos.

\subsubsection{Teorema do Ponto de Sela}

Teorema 2.2.1. [11] Seja $X$ um espaço de Banach e $V$ um subespaço de dimensão finita. Seja $W$ o complemento topológico de $V$, isto é, um subespaço de $X$ tal que $X=V \oplus W$. Seja $\phi: X \rightarrow \mathbb{R}$ um funcional $\mathcal{C}^{1}$ que satisfaz a condição $(P S)$. Suponha que existam constantes a e b e um número $R>0$, tais que

$$
\begin{gathered}
\left.\phi\right|_{\partial B_{R}(0) \cap V} \leq a \\
\left.\phi\right|_{W} \geq b \\
a<b
\end{gathered}
$$

onde $B_{R}(0)$ designa a bola de raio $R$ e centrada em 0 no espaço $X$. Então, $\phi$ tem um ponto crítico no nivel c definido por

$$
c=\inf _{\gamma \in \Gamma} \max _{u \in B_{R}(0) \cap V} \phi(\gamma(u))
$$

onde $\Gamma=\left\{\gamma: \overline{B_{R}(0)} \cap V \rightarrow X ; \gamma\right.$ é contínua, $\left.\gamma(u)=u, \forall u \in \partial B_{R}(0) \cap V\right\}$. 


\section{Demonstração:}

Suponhamos por contradição que $c$ não é valor crítico. Logo, existe $\varepsilon_{0}>0$ tal que $\phi$ não possui possui pontos críticos entre os níveis $c-\varepsilon_{0}$ e $c+\varepsilon_{0}$. De fato, se não existisse tal $\varepsilon_{0}$, teríamos uma sequência $\left\{u_{n}\right\}_{n} \subset X$ tal que

$$
\phi\left(u_{n}\right) \rightarrow c \quad \text { e } \quad \phi^{\prime}\left(u_{n}\right) \rightarrow 0 .
$$

Usando a condição $(P S)_{c}$, segue que existiria uma subsequência $\left\{u_{n_{j}}\right\}$ e $u_{0} \in X$ tais que $u_{n_{j}} \rightarrow u_{0}$. Como $\phi$ é de classe $\mathcal{C}^{1}$, obteríamos:

$$
\phi\left(u_{0}\right)=c \quad \text { e } \quad \phi^{\prime}\left(u_{0}\right)=0
$$

o que é um absurdo.

Tomemos agora $\bar{\varepsilon}<c-a$ e $\varepsilon<\varepsilon_{0}$. Pelo Lema 2.2.3, temos $c \geq b$. Tomemos $\varepsilon<\bar{\varepsilon}$, e seja $\gamma \in \Gamma$ de modo que

$$
\max _{u \in \overline{B_{R}(0)} \cap V} \phi(\gamma(u))<c+\varepsilon .
$$

Defina $\tilde{\gamma}(v)=\eta(1, \gamma(v))$, onde $\eta \in \mathcal{C}([0,1] \times X, X)$ é dado com no Lema de Deformação (Lema 2.2.2). Mostraremos então que $\tilde{\gamma} \in \Gamma$. A continuidade segue de forma clara. Resta-nos mostrar que para $v \in \partial B_{R}(0) \cap V$, temos $\tilde{\gamma}(v)=v$. Note que $\gamma(v)=v$ e $\phi(v) \leq a<c-\bar{\varepsilon}$, pois $\gamma \in \Gamma$ e tomamos $\bar{\varepsilon}$ de modo que tal desigualdade fosse válida. Assim, a conclusão (ii) do Lema 2.2.2 nos diz que

$$
\eta(1, v)=v
$$

isto é, $v=\eta(1, \gamma(v))=\tilde{\gamma}(v)$. Portanto,

$$
\tilde{\gamma}(v)=v
$$

e assim, $\tilde{\gamma} \in \Gamma$. Note agora que $\phi(\tilde{\gamma}(v))=\phi(\eta(1, \gamma(v)))$ e que $\phi(\gamma(v))<c+\varepsilon$.

Assim, segue-se do Lema 2.2.2, item (iii) que

$$
\underset{v \in \max _{B_{R}(0)} \cap V}{ } \phi(\tilde{\gamma}(v))<c-\varepsilon,
$$

o que contradiz o fato de $c$ ser o ínfimo.

\subsubsection{Demonstração do Teorema $\mathbf{0 . 0 . 2}$}

Dados $\lambda_{j}<\lambda_{j+1}$ dois autovalores consecutivos, designamos por $H_{j}$ o subespaço de $H_{0}^{1}(\Omega)$ gerado pelas autofunções correspondentes aos autovalores $\lambda_{i}$ com $i \leq j$. Deste modo, $H_{j}^{\perp}, \mathrm{o}$ complemento ortogonal de $H_{j}$ é gerado pelas autofunções correspondentes aos autovalores $\lambda_{i}$ com $i \geq j+1$. Note que $H_{j}$ tem dimensão finita. Pela equação (D.3) do Apêndice D, temos as seguintes desigualdades:

$$
\int_{\Omega}|\nabla v|^{2} d x \leq \lambda_{j} \int_{\Omega} v^{2} d x, \forall v \in H_{j}
$$




$$
\int_{\Omega}|\nabla w|^{2} d x \geq \lambda_{j+1} \int_{\Omega} w^{2} d x, \forall v \in H_{j}^{\perp} .
$$

Seguem-se de (2), (4) e da continuidade de $\mathrm{F}$ em $\bar{\Omega} \times \mathbb{R}$ que existem constantes $c_{1}, c_{2}, d_{1}$ e $d_{2}$ com $\lambda_{j}<c_{1}<c_{2}<\lambda_{j+1}$ e $d_{1}, d_{2}>0$ e tais que

$$
\frac{1}{2} c_{1} s^{2}-d_{1} \leq F(x, s) \leq \frac{1}{2} c_{2} s^{2}+d_{2} .
$$

Usando (2.12) obtemos para $v \in H_{j}$ :

$$
\begin{aligned}
\phi(v) & =\frac{1}{2} \int_{\Omega}|\nabla v|^{2} d x-\int_{\Omega} F(x, v) d x \\
& \leq \frac{1}{2} \int_{\Omega}|\nabla v|^{2} d x-\frac{1}{2} c_{1} \int_{\Omega} v^{2} d x+c,
\end{aligned}
$$

para alguma constante $c>0$. De (2.10), segue que

$$
\begin{aligned}
\phi(v) & \leq \frac{1}{2} \int_{\Omega}|\nabla v|^{2} d x-\frac{1}{2} \frac{c_{1}}{\lambda_{j}} \int_{\Omega}|\nabla v|^{2} d x+c \\
& =\frac{1}{2}\left(1-\frac{c_{1}}{\lambda_{j}}\right) \int_{\Omega}|\nabla v|^{2} d x+c .
\end{aligned}
$$

Como $c_{1}>\lambda_{j}$, segue que $\phi(v) \rightarrow-\infty$, quando $\|v\|_{H_{0}^{1}(\Omega)} \rightarrow \infty$. Analogamente, de (2.12) e (2.11) segue-se que para $w \in H_{j}^{\perp}$ :

$$
\begin{aligned}
\phi(w) & \geq \frac{1}{2} \int_{\Omega}|\nabla w|^{2} d x-\frac{1}{2} \frac{c_{2}}{\lambda_{j+1}} \int_{\Omega}|\nabla w|^{2} d x-c \\
& =\frac{1}{2}\left(1-\frac{c_{2}}{\lambda_{j+1}}\right) \int_{\Omega}|\nabla w|^{2} d x-c .
\end{aligned}
$$

Como $c_{2}<\lambda_{j+1}$, concluímos que

$$
\inf \left\{\phi(w) ; w \in H_{j}^{\perp}\right\}=b>-\infty .
$$

Escolha agora $R>0$ de modo que

$$
\sup \left\{\phi(v) ; v \in H_{j},\|v\|_{H_{0}^{1}(\Omega)}=R\right\}=: a<b<0 .
$$

Finalmente, como $\phi$ satisfaz $(P S)$ podemos aplicar o Teorema 1.2.3 e concluir a existência de um ponto crítico para o funcional $\phi$ e, consequentemente, uma solução de (1). 



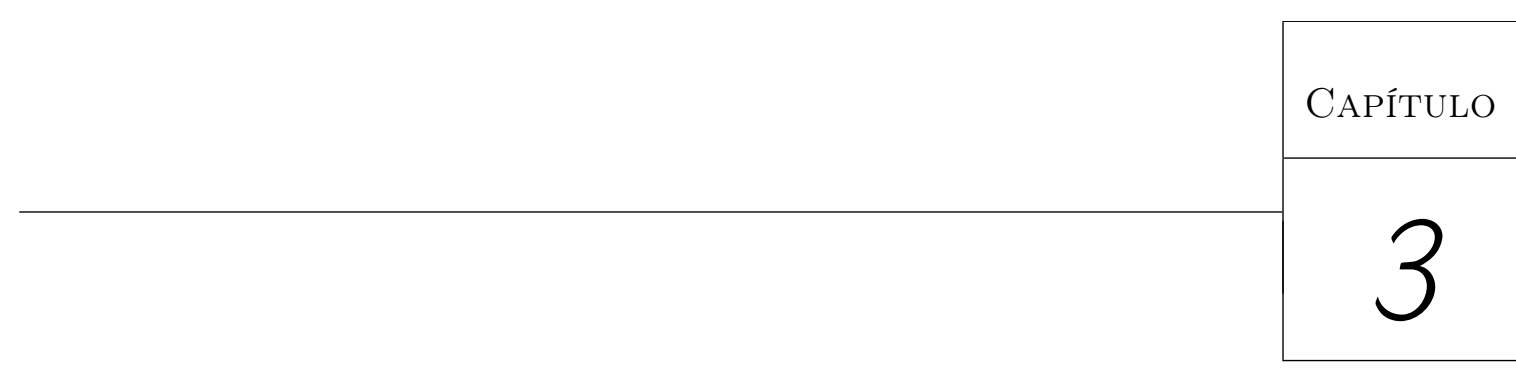

\section{Teorema 0.0 .3}

Para demonstrar o Teorema 0.0.3, utilizaremos o teorema do passo da montanha [11] enunciado a seguir.

\subsection{Teorema do Passo da Montanha}

Teorema 3.1.1. Seja $X$ um espaço de Banach e $\phi: X \rightarrow \mathbb{R}$ uma função de classe $\mathcal{C}^{1}$. Suponhamos que $\phi$ satisfaça as seguintes condições geométricas:

(H1) Existem $R>0$ e $c_{0} \in \mathbb{R}$ tais que $\phi(u) \geq c_{0}$ para todo $u \in S_{R}:=\{v \in X ;\|v\|=R\}$;

(H2) $\phi(0)<c_{0}$ e $\phi(e)<c_{0}$ para algum $e \in X$ com $\|e\|>R$.

Assim, se $\phi$ satisfizer a condição de Palais-Smale então $\phi$ terá um ponto crítico em $X \backslash\{0, e\}$ com correspondente valor crítico $c \geq c_{0}$.

\section{Demonstração:}

Definamos

$$
P=\{p \in \mathcal{C}([0,1], X) ; p(0)=0, p(1)=e\}
$$

Mostremos que

$$
c=\inf _{p \in P} \sup _{t \in[0,1]} \phi(p(t))
$$

é um valor crítico. 
Suponhamos, por absurdo, que $K_{c}=\left\{u \in X ; \phi^{\prime}(u)=0, \phi(u)=c\right\}=\emptyset$ e tomemos $\bar{\varepsilon}=\min \{c-\phi(0), c-\phi(e)\}$. Pela definição de $c, \bar{\varepsilon}>0$. Tomemos $\varepsilon \in(0, \bar{\varepsilon} / 2)$ e a deformação $\eta$ dada pelo Lema 2.2.2. Pela definição de $c$, existe $p \in P$ tal que

$$
\sup _{t \in[0,1]} \phi(p(t))<c+\varepsilon .
$$

Considere $p_{1}(t)=\eta(p(t), 1)$. Pela definição de $\bar{\varepsilon}$, para todo $\varepsilon \in(0, \bar{\varepsilon} / 2)$, temos

$$
\phi(0), \phi(e)<c-2 \varepsilon \text {. }
$$

Pelo Lema 2.2.2, $\eta(1,0)=0$ e $\eta(1, e)=e$, e portanto, $p_{1} \in P$. Mais que isto, $\eta\left(\phi_{c+\varepsilon}, 1\right) \in \phi_{c-\varepsilon}$, isto é,

$$
\sup _{t \in[0,1]} \phi\left(p_{1}(t)\right)=\sup _{t \in[0,1]} \phi(\eta(p(t), 1))<c-\varepsilon .
$$

Mas, isto contradiz a definição de $c$, completando a prova.

\subsection{Demonstração do Teorema $\mathbf{0 . 0 . 3}$}

Uma vez que estamos procurando soluções positivas é natural considerarmos a função

$$
f_{0}(u):=\left\{\begin{array}{l}
f(u), \text { se } u \geq 0 \\
0, \text { se } u<0
\end{array}\right.
$$

Tal função é contínua, pois $f: \mathbb{R} \rightarrow \mathbb{R}$ é contínua com $f(0)=0$. Pelas hipóteses (5)-(6), existe $C>0$ tal que para todo $u \in \mathbb{R}$,

$$
\left|f_{0}(u)\right| \leq C(1+|u|)
$$

Por (6), existem constantes $C_{1}>\lambda_{1}$ e $C_{2}>0$ tais que $f_{0}(u) \geq C_{1} u-C_{2}$, para todo $u$. Defina

$$
F_{0}(u)=\int_{0}^{u} f(t) d t
$$

Assim,

$$
\left|F_{0}(u)\right| \leq C\left(u+\frac{u^{2}}{2}\right)
$$

e

$$
F_{0}(u) \geq C_{1}^{\prime} \frac{u^{2}}{2}-C_{3}, \operatorname{com} C_{1}>C_{1}^{\prime}>\lambda_{1} .
$$

Associamos a (7) o funcional energia $\phi: H_{0}^{1}(\Omega) \rightarrow \mathbb{R}$ definido por

$$
\phi(u)=\frac{1}{2} \int_{\Omega}|\nabla u|^{2} d x-\int_{\Omega} F_{0}(u) d x, \forall u \in H_{0}^{1}(\Omega) .
$$

Segue de (3.1) e do Teorema das Imersões de Sobolev (Teorema A.1.1) que $\phi$ está bem definido em $H_{0}^{1}(\Omega)$. Mais que isto, $\phi$ é de classe $\mathcal{C}^{1}$ e para todo $v \in H_{0}^{1}(\Omega)$

$$
\phi^{\prime}(u) v=\int_{\Omega}\left[\nabla u \nabla v-f_{0}(u) v\right] d x .
$$


Mostraremos inicialmente que a geometria do Passo da Montanha é satisfeita. Fixemos $1<p<\frac{N+2}{N-2}$. Para verificar a condição (H1), notemos que (5) e (6) implicam que existem $0<\alpha<\lambda_{1}$ e $C_{4}>0$ tais que

$$
\left|f_{0}(s)\right| \leq \alpha|s|+C_{4}|s|^{p}, \quad \forall s \in \mathbb{R}
$$

Assim,

$$
F_{0}(s) \leq \alpha \frac{s^{2}}{2}+C_{5}|s|^{p+1}, C_{5}>0, \quad \forall s \in \mathbb{R}
$$

Segue então que

$$
\phi(u) \geq \frac{1}{2} \int_{\Omega}|\nabla u|^{2} d x-\frac{\alpha}{2} \int_{\Omega} u^{2} d x-C_{5} \int_{\Omega}|u|^{p+1} d x .
$$

Pela Desigualdade de Poincaré, temos:

$$
\int_{\Omega} u^{2} d x \leq \frac{1}{\lambda_{1}} \int_{\Omega}|\nabla u|^{2} d x, \forall u \in H_{0}^{1}(\Omega) .
$$

Segue então que

$$
\phi(u) \geq \frac{\lambda_{1}-\alpha}{2 \lambda_{1}}\|u\|_{H_{0}^{1}(\Omega)}^{2}-C_{5} \int_{\Omega}|u|^{p+1} d x, \forall u \in H_{0}^{1}(\Omega) .
$$

Utilizando as Imersões de Sobolev, segue que $\phi(u)>0$ para todo $u$ com $\|u\|_{H_{0}^{1}(\Omega)}=R$ e $R$ suficientemente pequeno. Assim, a condição (H1) é satisfeita, em particular, com $c_{0}>0$.

Provemos agora que (H2) ocorre. Seja $w_{1}$ a primeira autofunção de $-\Delta$ em $H_{0}^{1}(\Omega)$, isto é,

$$
\int_{\Omega}\left|\nabla w_{1}\right|^{2} d x=\lambda_{1} \int_{\Omega} w_{1}^{2} d x
$$

Deste modo, de (3.2) e do fato que $C_{1}>\lambda_{1}$, segue que

$$
\phi\left(t w_{1}\right) \leq \frac{t^{2} \lambda_{1}^{2}}{2} \int_{\Omega} w_{1}^{2} d x-\frac{t^{2} C_{1}^{2}}{2} \int_{\Omega} w_{1}^{2} d x+C_{3}|\Omega|<0,
$$

para todo $t>0$ suficientemente grande. Logo, a condição (H2) está satisfeita escolhendo $e=t w_{1}$ para este $t>0$ suficientemente grande.

Para completar a prova resta mostrar que o funcional $\phi$ satisfaz a condição (PS). Seja $\left\{u_{n}\right\}_{n}$ uma sequência em $H_{0}^{1}(\Omega)$ tal que

$$
\phi\left(u_{n}\right) \rightarrow c
$$

e

$$
\phi^{\prime}\left(u_{n}\right) \rightarrow 0
$$

onde $c$ é uma constante qualquer

Mostremos que

$$
\left\{u_{n}\right\} \text { é limitada em } H_{0}^{1}(\Omega) \text {. }
$$


Notemos inicialmente que a relação (3.5) nos diz que

$$
-\Delta u_{n}=f_{0}\left(u_{n}\right)+\psi_{n}, \text { onde }\left\|\psi_{n}\right\|_{H^{-1}(\Omega)} \rightarrow 0 .
$$

Combinando (3.3) e (3.7), deduzimos (3.6) desde que $\left\{u_{n}\right\}$ seja limitada em $L^{2}(\Omega)$. De fato, note que

$$
\phi^{\prime}\left(u_{n}\right) u_{n}=\int_{\Omega}\left|\nabla u_{n}\right|^{2} d x-\int_{\Omega} f\left(u_{n}\right) u_{n} d x
$$

implicando que

$$
\left\|u_{n}\right\|_{H_{0}^{1}(\Omega)}^{2}=\phi^{\prime}\left(u_{n}\right) u_{n}+\int_{\Omega} f_{0}\left(u_{n}\right) u_{n} d x
$$

Assim,

$$
\begin{aligned}
\left\|u_{n}\right\|_{H_{0}^{1}(\Omega)}^{2} & \leq\left\|\phi^{\prime}\left(u_{n}\right)\right\| \cdot\left\|u_{n}\right\|_{H_{0}^{1}(\Omega)}+\int_{\Omega}\left|f_{0}\left(u_{n}\right) u_{n}\right| d x \\
& \leq\left\|\phi^{\prime}\left(u_{n}\right)\right\| \cdot\left\|u_{n}\right\|_{H_{0}^{1}(\Omega)}+\alpha \int_{\Omega} u_{n}^{2} d x+C \int_{\Omega}\left(1+\left|u_{n}\right|\right)\left|u_{n}\right| d x \\
& \leq\left\|\phi^{\prime}\left(u_{n}\right)\right\| \cdot\left\|u_{n}\right\|_{H_{0}^{1}(\Omega)}+\frac{\alpha}{\lambda_{1}}\left\|u_{n}\right\|_{H_{0}^{1}(\Omega)}^{2}+C^{\prime}\left(\int_{\Omega}\left|u_{n}\right|^{2} d x\right)^{\frac{1}{2}}+C \int_{\Omega}\left|u_{n}\right|^{2} d x .
\end{aligned}
$$

E, deste modo,

$$
\left(1-\frac{\alpha}{\lambda_{1}}\right)\left\|u_{n}\right\|_{H_{0}^{1}(\Omega)}^{2} \leq\left\|\phi^{\prime}\left(u_{n}\right)\right\|\left\|u_{n}\right\|_{H_{0}^{1}(\Omega)}+C^{\prime}\left\|u_{n}\right\|_{L^{2}(\Omega)}+C\left\|u_{n}\right\|_{L^{2}(\Omega)}^{2} .
$$

A Desigualdade acima nos diz que se $\left\{u_{n}\right\}$ for limitada em $L^{2}(\Omega)$, então $\left\{u_{n}\right\}$ também será limitada em $H_{0}^{1}(\Omega)$.

Mostremos então que $\left\{u_{n}\right\}_{n}$ é limitada em $L^{2}(\Omega)$. Suponha, por contradição, que $\left\|u_{n}\right\|_{L^{2}(\Omega)} \rightarrow \infty$. Defina

$$
v_{n}=\frac{u_{n}}{\left\|u_{n}\right\|_{L^{2}(\Omega)}}
$$

Assim, $v_{n}$ satisfaz:

$$
-\Delta v_{n}=\frac{f_{0}\left(u_{n}\right)}{\left\|u_{n}\right\|_{L^{2}(\Omega)}}+\frac{\psi_{n}}{\left\|u_{n}\right\|_{L^{2}(\Omega)}} .
$$

Notemos agora que $f_{0}\left(u_{n}\right) /\left\|u_{n}\right\|_{L^{2}(\Omega)}$ é limitada em $L^{2}(\Omega)$. Assim, multiplicando a equação (3.8) por $v_{n}$ e integrando, obtemos que $\left\{v_{n}\right\}$ é limitada em $H_{0}^{1}(\Omega)$. Logo, existem uma subsequência de $\left\{v_{n}\right\}$, ainda denotada por $\left\{v_{n}\right\}$, e $v$ tais que

$$
v_{n} \rightarrow v \text { em } H_{0}^{1}(\Omega)
$$

E, como $\left\|v_{n}\right\|_{L^{2}(\Omega)}=1$, então

$$
\|v\|_{L^{2}(\Omega)}=1 .
$$

Observemos agora que a definição de $f_{0}$ implica que existe $c>0$ tal que $f_{0}(u) \geq-c$, para todo $u \in \mathbb{R}$. Assim, por (3.8),

$$
-\Delta u_{n} \geq-c+\psi_{n}, \quad x \in \Omega .
$$


Pelo princípio do máximo (Teorema B.0.6), $u_{n} \geq-c+\rho_{n}$, onde $\rho_{n} \rightarrow 0$ em $H_{0}^{1}(\Omega)$ e, assim, existe uma subsequência de $\left\{\rho_{n}\right\}$, também denotada por $\left\{\rho_{n}\right\}$, e tal que

$$
\rho_{n} \rightarrow 0 \text { quase sempre em } \Omega \text {. }
$$

E, isto implica que

$$
v \geq 0 \text { quase sempre em } \Omega \text {. }
$$

Por outro lado, a equação (6) implica que

$$
f_{0}(u) \geq A u-B, \forall u \in \mathbb{R},
$$

onde $A>\lambda_{1}$ e $B>0$. Isto implica que

$$
-\Delta u_{n} \geq A u_{n}-B+\psi_{n}, \quad x \in \Omega .
$$

Sendo $w_{1}>0$ a autofunção associada ao primeiro autovalor de $-\Delta$ em $H_{0}^{1}(\Omega)$, de (3.11), obtemos:

$$
\lambda_{1} \int_{\Omega} u_{n} \psi_{n} d x \geq A \int_{\Omega} u_{n} w_{1} d x-C+<\psi_{n}, w_{n}>_{H^{-1}, H_{0}^{1}} .
$$

Dividindo por $\left\|u_{n}\right\|_{L^{2}(\Omega)}$ e tomando $n \rightarrow \infty$, obtemos:

$$
\lambda_{1} \int_{\Omega} v w_{1} d x \geq A \int_{\Omega} v w_{1} d x
$$

que contradiz (3.9) e (3.10). Assim, fica provado (3.6).

Definamos agora $D: H_{0}^{1}(\Omega) \rightarrow H^{-1}$ por

$$
D(u) \varphi=\int_{\Omega} \nabla u \nabla \varphi d x .
$$

Pela Desigualdade de Hölder, a transformação linear $D$ é contínua.

Afirmamos que $D$ é bijetiva. Primeiramente, $D$ é injetiva pois $D(u) \varphi=0$ para todo $\varphi \in H_{0}^{1}(\Omega)$ se, e somente se, $u=0$. Para verificar que $D$ é sobrejetiva, notemos que $H^{-1} \subset L^{2}(\Omega)$ e assim para $f \in H^{-1}$ dado, pelo Apêndice D.1 existe um único $u \in H_{0}^{1}(\Omega)$ tal que $D(u)=f$. Deste modo, $D$ é uma transformação linear, bijetiva, contínua. Logo $D^{-1}$ também será uma tranformação contínua.

Agora, notemos que

$$
\phi^{\prime}(u) \varphi=\int_{\Omega} \nabla u \nabla \varphi d x-\int_{\Omega} f \varphi d x=D(u) \varphi-J^{\prime}(u) \varphi,
$$

isto é, $\phi^{\prime}(u)=D(u)-J^{\prime}(u)$ em $H^{-1}$. Logo, $D^{-1} \phi^{\prime}(u)=u-D^{-1} J^{\prime}(u)$. Assim, para a sequência $\left\{u_{n}\right\}$ limitada em $H_{0}^{1}(\Omega)$ :

$$
u_{n}=D^{-1} \phi^{\prime}\left(u_{n}\right)+D^{-1} J^{\prime}\left(u_{n}\right) .
$$

Uma vez que $D^{-1}$ é contínuo, $J^{\prime}$ é compacto e $\phi^{\prime}\left(u_{n}\right) \rightarrow 0$, então $\left\{u_{n}\right\}$ possui subsequência convergente, mostrando que $\phi$ satisfaz a condição de Palais-Smale.

Aplicando o Teorema do Passo da Montanha (Teorema 3.1.1), o funcional $\phi$ possui um ponto crítico em $H_{0}^{1}(\Omega) \backslash\left\{0, w_{1}\right\}$. Logo, o problema (7) possui uma solução fraca. 



\section{Espaços de Sobolev}

Neste apêndice resumiremos alguns resultados utilizados nesta dissertação, cujas demonstrações podem ser encontradas em [3] e [7].

Consideremos $\Omega \subset \mathbb{R}^{N}$ um domínio limitado. Seja $x \in \mathbb{R}^{N}$ com coordenadas $\left(x_{1}, \cdots, x_{N}\right)$. Um multi-índice é uma N-upla

$$
\alpha=\left(\alpha_{1}, \cdots, \alpha_{N}\right), \alpha_{i} \geq 0, \alpha_{i} \text { inteiros. }
$$

Associado ao multi-índice $\alpha$, temos

$$
|\alpha|=\alpha_{1}+\cdots+\alpha_{N}
$$

Definimos:

$$
D^{\alpha}=\frac{\partial^{|\alpha|}}{\partial x_{1}^{\alpha_{1}} \cdots \partial x_{N}^{\alpha_{N}}} .
$$

Denote por $\mathcal{C}_{c}^{\infty}(\Omega)=\mathcal{C}^{\infty}(\Omega) \cap \mathcal{C}_{c}(\Omega)$, onde $\mathcal{C}_{c}(\Omega)$ é o conjunto das funções contínuas com suporte compacto em $\Omega$.

Definição A.0.1. Seja $m>0$ um inteiro $e 1 \leq p \leq \infty$. O Espaço de Sobolev $W^{m, p}(\Omega)$ é definido por

$W^{m, p}(\Omega)=\left\{u \in L^{p}(\Omega) ; \forall|\alpha| \leq m, \exists g_{\alpha} \in L^{p}(\Omega) ; \int_{\Omega} u D^{\alpha} \varphi=(-1)^{|\alpha|} \int_{\Omega} g_{\alpha} \varphi, \forall \varphi \in \mathcal{C}_{c}^{\infty}(\Omega)\right\}$.

Denotemos $D^{\alpha} u:=g_{\alpha}$. 
O espaço definido acima é vetorial. Consideremos em tal espaço a seguinte norma:

$$
\|u\|_{m, p, \Omega}=\sum_{|\alpha| \leq m}\left\|D^{\alpha} u\right\|_{L^{p}(\Omega)} .
$$

Consideremos também a seguinte semi-norma:

$$
|u|_{m, p, \Omega}=\sum_{|\alpha|=m}\left\|D^{\alpha} u\right\|_{L^{p}(\Omega)}
$$

Definição A.0.2. Definamos o espaço $W_{0}^{m, p}(\Omega)$ como sendo o fecho de $\mathcal{C}_{c}^{\infty}(\Omega)$ em $W^{m, p}(\Omega)$.

\section{Notações:}

Quando $p=2$, escremos:

$$
\begin{aligned}
& W^{m, 2}(\Omega)=H^{m}(\Omega) \\
& W_{0}^{m, 2}(\Omega)=H_{0}^{m}(\Omega) .
\end{aligned}
$$

Teorema A.0.1. Para todo $1 \leq p \leq \infty$, o espaço $W^{m, p}(\Omega)$ é um espaço de Banach. Se $1<p<\infty$, este espaço é reflexivo e se $1 \leq p<\infty$, é separável. Em particular $H^{1}(\Omega)$ é um espaço de Hilbert separável.

Teorema A.0.2. (Desigualdade de Poincaré) Seja $\Omega$ um conjunto aberto e limitado em $\mathbb{R}^{N}$. Então existe uma constante positiva $C=C(\Omega, p)$ tal que

$$
\|u\|_{L^{p}(\Omega)} \leq C|u|_{1, p, \Omega}, \forall u \in W_{0}^{1, p}(\Omega) .
$$

Assim, $u \mapsto|u|_{1, p, \Omega}$ define uma norma em $W_{0}^{1, p}(\Omega)$, que é equivalente a norma $\|\cdot\|_{1, p, \Omega}$, e será denotada por $\|\cdot\|_{W_{0}^{1, p}(\Omega)}$. Além disso,

$$
\lambda_{1}(\Omega):=\inf \left\{\|u\|_{H_{0}^{1}(\Omega)}^{2} ; u \in H_{0}^{1}(\Omega),\|u\|_{L^{2}(\Omega)}=1\right\}>0 .
$$

é atingido.

\section{A.1 Teoremas de Imersão}

Teorema A.1.1. Seja $\Omega \subset \mathbb{R}^{N}$ um conjunto aberto e limitado de classe $\mathcal{C}^{1}$. Então as seguintes imersões são compactas:

(i) Se $1 \leq p<N, W^{1, p}(\Omega) \hookrightarrow L^{q}(\Omega), 1 \leq q<p^{*}$, onde $p^{*}=\frac{N p}{N-p}$;

(ii) Se $p=N, W^{1, N}(\Omega) \hookrightarrow L^{q}(\Omega), 1 \leq q<\infty$;

(iii) Se $p>N, W^{1, p}(\Omega) \hookrightarrow \mathcal{C}^{0, \alpha}(\bar{\Omega})$, onde $0<\alpha<1-\frac{N}{p}$.

Observação A.1.1. Se $k \geq 1$ for um inteiro tal que $k-\frac{N}{p}>0$, então a imersão $W^{1, p}(\Omega) \hookrightarrow \mathcal{C}^{m, \alpha}(\bar{\Omega})$ é contínua, onde $m=\left[k-\frac{N}{p}\right]$ e $\alpha=k-\frac{N}{p}-m$, e a imersão é compacta em $\mathcal{C}^{m, \beta}(\bar{\Omega})$ para qualquer $\beta<\alpha$. 


\section{Alguns Resultados}

As demonstrações dos teoremas enunciados neste apêndice podem ser encontradas em [7].

Teorema B.0.2. Seja E um espaço de Banach. Então E é reflexivo se, e somente se,

$$
B_{E}=\{x \in E ;\|x\| \leq 1\}
$$

for compacta na topologia fraca.

Teorema B.0.3. Sejam $\Omega \subset \mathbb{R}^{N}$ um dominio limitado com fronteira suave, $f \in L^{r}(\Omega)$ com $r>1$ e $u \in H_{0}^{1}(\Omega)$ uma solução fraca do problema

$$
\left\{\begin{array}{l}
-\Delta u=f(x), \quad x \in \Omega, \\
u=0, \quad x \in \partial \Omega .
\end{array}\right.
$$

Então, $u \in W^{2, r}(\Omega)$ e existe $C>0$ tal que

$$
\|u\|_{W^{2, r}(\Omega)} \leq C\|f\|_{L^{r}(\Omega)} .
$$

Observação B.0.2. O Teorema acima nos diz que se $f \in W^{k, r}(\Omega)$, então $u \in W^{k+2, r}(\Omega)$.

Teorema B.0.4. Seja $\Omega \subset \mathbb{R}^{N}$ um dominio limitado com fronteira suave e $f \in \mathcal{C}^{0, \alpha}(\bar{\Omega})$. Então existe $u \in \mathcal{C}^{2, \alpha}(\bar{\Omega})$ solução do problema

$$
\left\{\begin{array}{l}
-\Delta u=f(x), \quad x \in \Omega, \\
u=0, \quad x \in \partial \Omega .
\end{array}\right.
$$


Além disso, existe $C>0$ tal que

$$
\|u\|_{\mathcal{C}^{2, \alpha}(\bar{\Omega})} \leq C\|f\|_{\mathcal{C}^{0, \alpha}(\bar{\Omega})}
$$

Observação B.0.3. O Teorema acima nos diz que se $f \in \mathcal{C}^{k, \alpha}(\bar{\Omega})$, então $u \in \mathcal{C}^{k+2, \alpha}(\bar{\Omega})$.

Teorema B.0.5. (Alternativa de Fredholm) Seja T uma aplicação linear e compacta de um espaço vetorial normado $\mathcal{V}$ nele mesmo. Então apenas uma das duas afirmações ocorre:

(i) A equação homogênea

$$
x-T x=0
$$

possui uma solução não-trivial $x \in \mathcal{V}$.

(ii) Para cada $y \in \mathcal{V}$, a equação

$$
x-T x=y
$$

possui uma única solução $x \in \mathcal{V}$.

Além disso, no caso de (ii), o operador $(I-T)^{-1}$ é limitado.

A demonstração do próximo resultado pode ser encontrada em [9].

Teorema B.0.6. (Princípio do Máximo) Sejam $a_{i j}, 1 \leq i, j \leq N$, e $a_{0}$ funções em $L^{\infty}(\Omega)$ tais que $a_{0} \geq 0$ e que $\left\{a_{i j}\right\}$ verifiquem

$$
\sum_{i, j=1}^{N} a_{i j}(x) \xi_{i} \xi_{j} \geq \alpha\left|\xi^{2}\right|, \forall \xi \in \mathbb{R}^{N}, x \in \Omega \text { onde } \alpha>0 .
$$

Seja $\Omega$ um conjunto aberto e limitado de $\mathbb{R}^{N}$ com fronteira suave $\partial \Omega$. Seja $f \in L^{2}(\Omega)$ e $u \in H^{1}(\Omega) \cap \mathcal{C}(\bar{\Omega})$ tal que

$$
\int_{\Omega} \sum_{i, j=1}^{N} a_{i j} \frac{\partial u}{\partial x_{j}} \frac{\partial v}{\partial x_{i}} d x+\int_{\Omega} a_{0} u v d x=\int_{\Omega} f v d x, \forall v \in H_{0}^{1}(\Omega) .
$$

Então:

(i) Se $f \geq 0$ em $\Omega$ e $u \geq 0$ em $\partial \Omega$ então $u \geq 0$ em $\Omega$.

(ii) Se $a_{0}=0$ e $f \geq 0$, então

$$
u \geq \inf _{\partial \Omega} u, e m \Omega .
$$

(iii) Se $f=0$ e $a_{0}=0$, então

$$
\inf _{\partial \Omega} u \leq u \leq \sup _{\partial \Omega} u .
$$




\section{O Funcional Energia}

Sob a hipótese que $f \in \mathcal{C}(\bar{\Omega} \times \mathbb{R}, \mathbb{R})$ e que satisfaz a condição (2) existe uma constante $C>0$ tal que

$$
|f(x, s)| \leq C(|s|+1), \forall x \in \bar{\Omega}, \forall s \in \mathbb{R} .
$$

Assim, fixado $1 \leq p<2^{*}$, podemos supor que

$$
|f(x, s)| \leq C\left(|s|^{p}+1\right) .
$$

Mostraremos no Teorema C.0.7 que o funcional $\phi: H_{0}^{1}(\Omega) \rightarrow \mathbb{R}$ dado por

$$
\phi(u)=\int_{\Omega}\left(\frac{1}{2}|\nabla u|^{2}-F(x, u)\right) d x, \quad \text { onde } \quad F(x, s)=\int_{0}^{s} f(x, t) d t,
$$

está bem definido, $\phi \in \mathcal{C}^{1}\left(H_{0}^{1}, \mathbb{R}\right)$ e que

$$
\phi^{\prime}(u) \varphi=\int_{\Omega}(\nabla u \cdot \nabla \varphi-f(x, u) \varphi) d x
$$

para todo $\varphi \in E=H_{0}^{1}(\Omega)$. Além disso, mostraremos que o funcional $J(u)=\int_{\Omega} F(x, u(x)) d x$ é sequencialmente fracamente contínuo e que $J^{\prime}(u)$ é compacto.

Proposição C.0.1. Seja $\Omega \subset \mathbb{R}^{N}$ um domínio limitado e seja g satisfazendo

$\left(g_{1}\right) g \in \mathcal{C}(\bar{\Omega} \times \mathbb{R}, \mathbb{R})$;

$\left(g_{2}\right)$ Existem constantes $r, s \geq 1$ e $a_{1}, a_{2} \geq 0$ tais que

$$
|g(x, \xi)| \leq a_{1}+a_{2}|\xi|^{\frac{r}{s}}, \forall x \in \bar{\Omega}, \xi \in \mathbb{R} .
$$


Então a aplicação

$$
\begin{aligned}
& T: L^{r}(\Omega) \rightarrow L^{s}(\Omega) \\
& \varphi \quad \mapsto g(\cdot, \varphi(\cdot))
\end{aligned}
$$

está em $\mathcal{C}\left(L^{r}(\Omega), L^{s}(\Omega)\right)$.

\section{Demonstração:}

Seja $u \in L^{r}(\Omega)$, então

$$
\int_{\Omega}|g(x, u(x))|^{s} d x \leq \int_{\Omega}\left(a_{1}+a_{2}|u(x)|^{\frac{r}{s}}\right)^{s} d x \leq a_{3} \int_{\Omega}\left(1+|u(x)|^{r}\right) d x<\infty .
$$

Portanto, $T: L^{r}(\Omega) \rightarrow L^{s}(\Omega)$. Para mostrar a continuidade de tal aplicação, observe que ela será contínua em $\varphi$ se, e somente se, a aplicação $z \mapsto f(x, z(x))$, onde

$$
f(x, z(x))=g(x, z(x)+\varphi(x))-g(x, \varphi(x)),
$$

for contínua em $z=0$. Portanto podemos admitir que $g(x, 0) \equiv 0$.

Seja $\varepsilon>0$ dado. Por $\left(g_{1}\right)$ e pelo fato que $g(x, 0)=0$, dado $\hat{\varepsilon}>0$, existe $\hat{\delta}>0$ tal que

$$
|g(x, \xi)| \leq \hat{\varepsilon}, \text { para } x \in \bar{\Omega} \text { e }|\xi| \leq \hat{\delta}
$$

Seja agora $u \in L^{r}(\Omega)$ com $\|u\|_{L^{r}(\Omega)} \leq \delta, \delta$ sendo livre por enquanto. Definamos

$$
\Omega_{1}=\{x \in \bar{\Omega} ;|u(x)| \leq \beta\} .
$$

Assim,

$$
\int_{\Omega_{1}}|g(x, u(x))|^{s} d x \leq \hat{\varepsilon}^{s}\left|\Omega_{1}\right| \leq \hat{\varepsilon}^{s}|\Omega| .
$$

Escolha $\hat{\varepsilon}$ de modo que

$$
\hat{\varepsilon}^{s}|\Omega| \leq\left(\frac{\varepsilon}{2}\right)^{s}
$$

Consideremos também $\Omega_{2}=\bar{\Omega} \backslash \Omega_{1}$. Como em (C.3), temos

$$
\int_{\Omega_{2}}|g(x, u(x))|^{s} d x \leq a_{3}\left(\left|\Omega_{2}\right|+\delta^{r}\right) .
$$

Mais que isto,

$$
\delta^{r} \geq \int_{\Omega_{2}}|u|^{r} d x \geq \beta^{r}\left|\Omega_{2}\right|
$$

Combinando as equações (C.5) e (C.6), obtemos:

$$
\int_{\Omega_{2}}|g(x, u(x))|^{s} d x \leq a_{3}\left(1+\beta^{-r}\right) \delta^{r} .
$$

Escolha $\delta$ de modo que

$$
a_{3}\left(1+\beta^{-r}\right) \delta^{r} \leq\left(\frac{\varepsilon}{2}\right)^{s} .
$$

E, assim, (C.4) e (C.7) implicam que

$$
\|g(\cdot, u)\|_{L^{s}(\Omega)} \leq \varepsilon \quad \text { se }\|u\|_{L^{r}(\Omega)}<\delta,
$$

o que completa a prova. 
Teorema C.0.7. Seja $\Omega$ um dominio suave em $\mathbb{R}^{N}$ cuja fronteira seja uma variedade suave e seja $f$ satisfazendo:

$\left(f_{1}\right) f(x, s) \in \mathcal{C}(\bar{\Omega} \times \mathbb{R}, \mathbb{R})$;

$\left(f_{2}\right)$ Existem $a_{1}, a_{2}>0$ constantes, tais que $|f(x, s)| \leq a_{1}+a_{2}|s|^{p}$, onde $0 \leq p<2^{*}-1 e$ $N \geq 3$.

Se

$$
F(x, s)=\int_{\Omega} f(x, t) d t \quad e \quad \phi(u)=\int_{\Omega}\left(\frac{1}{2}|\nabla u|^{2}-F(x, u)\right) d x,
$$

então $\phi \in \mathcal{C}^{1}\left(H_{0}^{1}, \mathbb{R}\right) e$

$$
\phi^{\prime}(u) \varphi=\int_{\Omega}(\nabla u \cdot \nabla \varphi-f(x, u) \varphi) d x,
$$

para todo $\varphi \in E=H_{0}^{1}(\Omega)$. Além disso, $J(u)=\int_{\Omega} F(x, u(x)) d x$ é sequencialmente fracamente contínua e $J^{\prime}(u)$ é compacta.

Observação C.0.4. Se $N=1$, a condição $\left(f_{2}\right)$ pode ser eliminada. Enquanto que para $N=2$, é suficiente supor que

$$
|f(x, s)| \leq a_{1} e^{\varphi(s)}
$$

onde

$$
\lim _{s \rightarrow 0} \frac{\varphi(s)}{s^{2}}=0 .
$$

No entanto, nesta dissertação restringiremos nossa atenção ao caso $N \geq 3$.

\section{Demonstração:}

Pelo Teorema A.1.1 e as hipóteses $\left(f_{1}\right)$ e $\left(f_{2}\right)$, $\phi$ está definida em $E$ e o mesmo é verdade para $\phi^{\prime}(u)$ com o cuidado da desigualdade de Hölder. Notemos que o primeiro termo de $\phi$ é $\mathcal{C}^{1}$ e sua derivada de Frechét é o primeiro termo de $\phi^{\prime}$. Assim, devemos mostrar que $J(u)=\int_{\Omega} F(x, u(x)) d x$ está em $\mathcal{C}^{1}(E, \mathbb{R})$.

Mostremos primeiramente que $J$ é Frechét diferenciável em $E$. Sejam $u, \varphi \in E$. Queremos mostrar que: dado $\varepsilon>0$, existe $\delta=\delta(\varepsilon, u)$ tal que

$$
\left|J(u+\varphi)-J(u)-\int_{\Omega} f(x, u) \varphi d x\right| \leq \varepsilon\|\varphi\|_{H_{0}^{1}(\Omega)}
$$

desde que $\|\varphi\|_{H_{0}^{1}(\Omega)} \leq \delta$.

Se o fato acima ocorrer, $J$ será Frechét diferenciável em $u$ e $J^{\prime}$ será dado pelo termo apropriado em (C.9). Definindo $\psi=|F(x, u(x)+\varphi(x))-F(x, u(x))-f(x, u(x)) \varphi(x)|$, temos:

$$
\left|J(u+\varphi)-J(u)-\int_{\Omega} f(x, u) \varphi d x\right| \leq \int_{\Omega} \psi d x .
$$

Definamos:

$$
\Omega_{1}=\{x \in \bar{\Omega} ;|u(x)| \geq \beta\}
$$




$$
\begin{gathered}
\Omega_{2}=\{x \in \bar{\Omega} ;|\varphi(x)| \geq \gamma\} \text { e } \\
\Omega_{3}=\{x \in \bar{\Omega} ;|u(x)| \leq \beta \text { e }|\varphi(x)| \leq \gamma\},
\end{gathered}
$$

com $\beta$ e $\gamma$ livres por enquanto. Deste modo,

$$
\int_{\Omega} \psi d x \leq \sum_{i=1}^{3} \int_{\Omega_{i}} \psi d x .
$$

Pelo Teorema do Valor Médio,

$$
F(x, s+\eta)-F(x, s)=f(x, s+\theta \eta) \eta, \text { onde } \theta \in(0,1) .
$$

Agora, por (C.12), a hipótese $\left(f_{2}\right)$ e a desigualdade de Hölder,

$$
\begin{aligned}
& \int_{\Omega_{1}}|F(x, u(x)+\varphi(x))-F(x, u(x))| d x \\
& =\int_{\Omega_{1}}|f(x, u(x)+\theta \varphi(x)) \varphi(x)| d x \\
& \leq \int_{\Omega_{1}}\left(a_{1}+a_{2}|u(x)+\theta \varphi(x)|^{p}\right)|\varphi(x)| d x \\
& \leq \int_{\Omega_{1}}\left[a_{1}-a_{2}(|u(x)|+\theta|\varphi|)^{p}\right]|\varphi(x)| d x \\
& \leq \int_{\Omega_{1}}\left[a_{1}+a_{2}(|u(x)|+|\varphi(x)|)^{p}\right]|\varphi(x)| d x \\
& \leq a_{1}\left|\Omega_{1}\right|^{\frac{N+2}{2 N}}\|\varphi\|_{L^{\frac{2 N}{N-2}(\Omega)}}+a_{2}\left|\Omega_{1}\right|^{\frac{1}{\sigma}}\left[\|u\|_{L^{p+1}(\Omega)}^{p}+\|\varphi\|_{L^{p+1}(\Omega)}^{p}\right]\|\varphi\|_{L^{\frac{2 N}{N-2}}(\Omega)},
\end{aligned}
$$

onde

$$
\frac{1}{\sigma}+\frac{p}{p+1}+\frac{N-2}{2 N}=1
$$

Observe que $p<\frac{N+2}{N-2}$ implica

$$
p+1<\frac{N+2}{N-2}+1=\frac{N+2+N-2}{N-2}=\frac{2 N}{N-2} .
$$

Assim,

$$
\frac{p}{p+1}<\frac{N+2}{N-2} \frac{N-2}{2 N}=\frac{N+2}{2 N}=1-\frac{(N-2)}{2 N} .
$$

Logo

$$
\frac{p}{p+1}+\frac{N-2}{2 N}<1
$$

Deste modo, existe $\sigma>1$ satisfazendo (C.14) e justificando (C.13). De (A.1) e (C.13), obtemos:

$\int_{\Omega_{1}}|F(x, u(x)+\varphi(x))-F(x, u(x))| d x \leq a_{4}\|\varphi\|_{H_{0}^{1}(\Omega)}\left[\left|\Omega_{1}\right|^{\frac{N+2}{2 N}}+\left|\Omega_{1}\right|^{\frac{1}{\sigma}}\left(\|u\|_{H_{0}^{1}(\Omega)}^{p}+\|\varphi\|_{H_{0}^{1}(\Omega)}^{p}\right)\right]$. 
Similarmente,

$$
\int_{\Omega_{1}}|f(x, u(x)) \varphi(x)| d x \leq a_{5}\|\varphi\|_{H_{0}^{1}(\Omega)}\left[\left|\Omega_{1}\right|^{\frac{n+2}{2 n}}+\left|\Omega_{1}\right| \frac{1}{\sigma}\|u\|_{H_{0}^{1}(\Omega)}^{p}\right] .
$$

Por (A.1) e a desigualdade de Hölder:

$$
\begin{gathered}
\|u\|_{H_{0}^{1}(\Omega)} \geq a_{6}\|u\|_{L^{2}(\Omega)} \geq a_{6}\|u\|_{L^{2}\left(\Omega_{1}\right)}=a_{6}\left(\int_{\Omega_{1}}|u|^{2}\right)^{\frac{1}{2}} \\
\geq a_{6}\left(\int_{\Omega_{1}} \beta^{2}\right)^{\frac{1}{2}}=a_{6}\left(\beta^{2}\right)^{\frac{1}{2}}\left|\Omega_{1}\right|^{\frac{1}{2}}=a_{6} \beta\left|\Omega_{1}\right|^{\frac{1}{2}} .
\end{gathered}
$$

Deste modo,

$$
\left|\Omega_{1}\right|^{\frac{1}{\sigma}} \leq\left(\frac{\|u\|_{H_{0}^{1}(\Omega)}}{a_{6} \beta}\right)^{\frac{2}{\sigma}}=: M_{1}, \quad\left|\Omega_{1}\right|^{\frac{n+2}{2 n}} \leq\left(\frac{\|u\|_{H_{0}^{1}(\Omega)}}{a_{6} \beta}\right)^{\frac{n+2}{n}}=: M_{2} .
$$

Note que $M_{1}, M_{2} \rightarrow 0$ quando $\beta \rightarrow \infty$. Combinando as equações (C.13)-(C.18):

$$
\begin{aligned}
& \int_{\Omega_{1}} \psi \leq a_{4}\|\varphi\|_{H_{0}^{1}(\Omega)}\left[\left|\Omega_{1}\right|^{\frac{N+2}{2 N}}+\left|\Omega_{1}\right|^{\frac{1}{\sigma}}\left(\|u\|_{H_{0}^{1}(\Omega)}^{p}+\|\varphi\|_{H_{0}^{1}(\Omega)}^{p}\right)\right] \\
& +a_{5}\|\varphi\|_{H_{0}^{1}(\Omega)}\left[\left|\Omega_{1}\right|^{\frac{N+2}{2 n}}+\left|\Omega_{1}\right|^{\frac{1}{\sigma}}\|u\|_{H_{0}^{1}(\Omega)}^{p}\right] \\
& \leq a_{4}\|\varphi\|_{H_{0}^{1}(\Omega)}\left[M_{2}+M_{1}\left(\|u\|_{H_{0}^{1}(\Omega)}^{p}+\|\varphi\|_{H_{0}^{1}(\Omega)}^{p}\right)\right]+a_{5}\|\varphi\|_{H_{0}^{1}(\Omega)}\left[M_{2}+M_{1}\|u\|_{H_{0}^{1}(\Omega)}^{p}\right] \\
& \leq a_{7}\left[M_{2}+M_{1}\left(\|u\|_{H_{0}^{1}(\Omega)}^{p}+\|\varphi\|_{H_{0}^{1}(\Omega)}^{p}\right]\|\varphi\|_{H_{0}^{1}(\Omega)} .\right.
\end{aligned}
$$

Podemos supor que $\delta \leq 1$. Deste modo, escolhemos $\beta$ suficientemente grande de modo que

$$
a_{7}\left[M_{2}+M_{1}\left(\|u\|_{H_{0}^{1}(\Omega)}^{p}+1\right)\right] \leq \frac{\varepsilon}{3} .
$$

Assim,

$$
\int_{\Omega_{1}} \psi d x<\frac{\varepsilon}{3}\|\varphi\|
$$

Similarmente, temos:

$$
\begin{aligned}
\int_{\Omega_{2}} \psi d x & \leq a_{3} \int_{\Omega_{2}}\left[1+(|u(x)|+|\varphi(x)|)^{p}|\varphi(x)|\right] d x \\
& \leq a_{4}\left(\int_{\Omega_{2}}\left(1+(|u(x)|+|\varphi(x)|)^{p}\right)^{\frac{p+1}{p}} d x\right)^{\frac{p}{p+1}}\|\varphi\|_{L^{p+1}\left(\Omega_{2}\right)} \\
& =a_{4}\left\|1+(u+\varphi)^{p}\right\|_{L^{\frac{p+1}{p}}\left(\Omega_{2}\right)}\left(\int_{\Omega_{2}}|\varphi|^{p+1} d x\right)^{\frac{1}{p+1}} \\
& \leq a_{5}\left\|1+(u+\varphi)^{p}\right\|_{H_{0}^{1}(\Omega)}\left(\int_{\Omega_{2}}|\varphi|^{p+1} d x\right)^{\frac{1}{p+1}} \\
& \leq a_{5}\left(1+\|u+\varphi\|_{H_{0}^{1}(\Omega)}^{p}\right)\left(\int_{\Omega_{2}}|\varphi|^{p+1} d x\right)^{\frac{1}{p+1}} \\
& \leq a_{5}\left(1+\|u\|_{H_{0}^{1}(\Omega)}^{p}+\|\varphi\|_{H_{0}^{1}(\Omega)}^{p}\right)\left(\int_{\Omega_{2}}|\varphi|^{p+1} d x\right)^{\frac{1}{p+1}} \\
& \leq a_{5}\left(1+\|u\|_{H_{0}^{1}(\Omega)}^{p}+\|\varphi\|_{H_{0}^{1}(\Omega)}^{p}\right)\left(\int_{\Omega_{2}}|\varphi(x)|^{p+1}\left(\frac{|\varphi(x)|}{\delta}\right)^{m-(p+1)} d x\right)^{\frac{1}{p+1}},
\end{aligned}
$$


onde $m=\frac{2 N}{N-2}>p+1$.

Observação C.0.5. Note que $|\varphi(x)| \geq \gamma$, para todo $x \in \Omega_{2}$. Assim,

$$
\frac{|\varphi(x)|}{\gamma} \geq 1
$$

Logo,

$$
\left(\frac{|\varphi(x)|}{\gamma}\right)^{m-(p+1)} \geq 1
$$

E, portanto,

$$
|\varphi(x)|^{p+1} \leq\left(\frac{\varphi(x)}{\gamma}\right)^{m-(p+1)}|\varphi(x)|^{m-(p+1)}
$$

Deste modo,

$$
\int_{\Omega_{2}} \psi d x \leq a_{6} \gamma^{\frac{p+1-m}{p+1}}\left(1+\|u\|^{p}+\|\varphi\|^{p}\right)\|\varphi\|^{\frac{m}{p+1}} .
$$

Agora, como $F \in \mathcal{C}^{1}(\bar{\Omega} \times \mathbb{R}, \mathbb{R})$, dados $\hat{\varepsilon}, \hat{\beta}>0$, existe $\hat{\gamma}=\hat{\gamma}(\hat{\varepsilon}, \hat{\beta})$ tal que

$$
|F(x, s+h)-F(x, s)-f(x, s) h|<\hat{\varepsilon}|h|,
$$

quando $x \in \bar{\Omega},|s| \leq \hat{\beta},|h|<\hat{\varepsilon}$. Em particular, se $\hat{\beta}$ e $\gamma \leq \hat{\gamma}$, a Desigualdade de Poincaré (A.1) e equação (C.21), implicam que:

$$
\int_{\Omega_{3}} \psi d x \leq \hat{\varepsilon} \int_{\Omega_{3}}|\varphi(x)| d x \leq a_{7} \hat{\varepsilon}\|\varphi\|_{H_{0}^{1}(\Omega)} .
$$

Escolha $\hat{\varepsilon}$ de modo que $3 a_{7} \hat{\varepsilon}<\varepsilon$. E, uma vez que $\hat{\gamma}=\hat{\gamma}(\hat{\varepsilon}, \hat{\beta})$, isto determina $\hat{\gamma}$. Combinando (C.11), (C.19), (C.20) e (C.22), temos:

$$
\int_{\Omega} \psi d x \leq \frac{2 \varepsilon}{3}\|\varphi\|_{H_{0}^{1}(\Omega)}+a_{6} \gamma^{\frac{p+1-m}{p+1}}\left(1+\|u\|_{H_{0}^{1}(\Omega)}^{p}+\|\varphi\|_{H_{0}^{1}(\Omega)}^{p}\right)\|\varphi\|_{H_{0}^{1}(\Omega)}^{\frac{m}{p+1}} .
$$

Finalmente, escolhemos $\delta$ suficientemente pequeno tal que

$$
a_{6} \gamma^{\frac{1-m}{p+1}}\left(2+\|u\|_{H_{0}^{1}(\Omega)}^{p}\right) \delta^{\frac{m}{p+1}-1} \leq \frac{\varepsilon}{3}
$$

e assim, obtemos (C.10).

Para provar que $J^{\prime}(u)$ é contínua, seja $u_{m} \rightarrow u$ em E. Então, por (A.1), $u_{m} \rightarrow u$ em $L^{p+1}(\Omega)$. Novamente pela Desigualdade de Poincaré e pela Desigualdade de Hölder,

$$
\begin{aligned}
\left\|J^{\prime}\left(u_{m}\right)-J^{\prime}(u)\right\| & =\sup _{\|\varphi\|_{H_{0}^{1}(\Omega)} \leq 1}\left|\int_{\Omega}\left(f\left(x, u_{m}(x)\right)-f(x, u(x))\right) \varphi(x) d x\right| \\
& \leq \sup _{\|\varphi\|_{H_{0}^{1}(\Omega)} \leq 1}\left\{\left\|f\left(\cdot, u_{m}\right)-f(\cdot, u)\right\|_{L^{\frac{p+1}{p}}(\Omega)}\|\varphi\|_{L^{p+1}(\Omega)}\right\} \\
& \leq \sup _{\|\varphi\|_{H_{0}^{1}(\Omega)} \leq 1}\left\{\left\|f\left(\cdot, u_{m}\right)-f(\cdot, u)\right\|_{L^{\frac{p+1}{p}}(\Omega)} a_{7}\|\varphi\|_{H_{0}^{1}(\Omega)}\right\} \\
& \leq a_{7}\left\|f\left(\cdot, u_{m}\right)-f(\cdot, u)\right\|_{L^{\frac{p+1}{p}}(\Omega)} .
\end{aligned}
$$


Por $\left(f_{2}\right)$, sabemos que $|f(x, s)| \leq a_{1}+a_{2}|s|^{\frac{\alpha p}{\alpha}}$, para algum $\alpha \geq 1$ e $\forall x \in \bar{\Omega}, s \in \mathbb{R}$. Pela Proposição C.0.1, $f \in \mathcal{C}\left(L^{\alpha p}(\Omega), L^{\alpha}(\Omega)\right)$. Tomando-se $\alpha=(p+1) / p$, o lado direito da equação (C.23) tende a zero quando $m \rightarrow \infty$ e $J^{\prime}$ é contínua.

Para provar que $J$ é sequencialmente fracamente contínuo, seja $\left\{u_{m}\right\}$ um sequência em $E$ convergindo fracamente a $u$. Pelo Teorema A.1.1, $u_{m}$ converge a $u$ em $L^{p+1}(\Omega)$, pois

$$
p+1<\frac{2 N}{N-2}=2^{*} .
$$

Consequentemente a proposição (C.0.1) implica que $J\left(u_{m}\right) \rightarrow J(u)$. Portanto, $J$ é fracamente contínuo em E. Para verificar a compacidade de $J^{\prime}(u)$, seja $\left\{u_{n}\right\}$ uma sequência limitada em $E$. A menos de subsequência, $u_{n} \rightarrow u$ para algum $u \in E$. Usando a imersão de Sobolev, $u_{n} \rightarrow u$ em $L^{p+1}(\Omega)$. Combinando (C.23) com a Proposição C.0.1 a conclusão se segue. 



\section{Espectro do Laplaciano}

$\mathrm{Na}$ demonstração dos teoremas enunciados na introdução, objetos deste estudo, fazemos uso do espectro do Laplaciano sob condição de Dirichlet, isto é, os números $\lambda \in \mathbb{R}$ tais que o problema

$$
\left\{\begin{array}{l}
-\Delta u=\lambda u, \quad x \in \Omega, \\
u=0, \quad x \in \partial \Omega
\end{array}\right.
$$

possui uma solução não trivial. Para desenvolver tal estudo necessitaremos de alguns resultados que serão desenvolvidos a seguir.

\section{D.1 O operador solução}

Dado $f \in L^{2}(\Omega)$, afirmamos que existe uma única solução $u \in H_{0}^{1}(\Omega)$ do problema

$$
\left\{\begin{array}{l}
-\Delta u=f, \quad x \in \Omega, \\
u=0, \quad x \in \partial \Omega .
\end{array}\right.
$$

De fato, dado $f \in L^{2}(\Omega)$, defina o funcional linear

$$
\begin{aligned}
F: H_{0}^{1}(\Omega) & \longrightarrow \mathbb{R} \\
\phi & \longmapsto F(\phi)=\int_{\Omega} f \phi d x .
\end{aligned}
$$

Pelas desigualdades de Hölder e de Poincaré, temos

$$
|F(\phi)| \leq\|f\|_{L^{2}(\Omega)}\|\phi\|_{L^{2}(\Omega)} \leq C\|f\|_{L^{2}(\Omega)}\|\phi\|_{H_{0}^{1}(\Omega)},
$$


o que implica,

$$
|F(\phi)| \leq M\|\phi\|_{H_{0}^{1}(\Omega)} \quad ; \quad M=C\|f\|_{L^{2}(\Omega)}
$$

mostrando a continuidade de $F$, ou seja, $F \in H^{-1}(\Omega)$, o espaço dual $H_{0}^{1}(\Omega)$. Pelo Teorema da Representação de Riesz, existe um único $u \in H_{0}^{1}(\Omega)$ tal que

$$
(u, \phi)_{H_{0}^{1}(\Omega)}=F(\phi), \quad \forall \phi \in H_{0}^{1}(\Omega)
$$

de onde segue

$$
\int_{\Omega} \nabla u \nabla \phi d x=\int_{\Omega} f \phi d x, \forall \phi \in H_{0}^{1}(\Omega)
$$

ou seja, $u$ é a única solução fraca do problema $\left(P_{1}\right)$.

Pelo que foi visto até o momento, podemos definir o operador solução dado por

$$
\begin{aligned}
S: L^{2}(\Omega) & \longrightarrow H_{0}^{1}(\Omega) \\
f & \longmapsto S(f)=u,
\end{aligned}
$$

que associa a cada $f \in L^{2}(\Omega)$ a única solução $u=S(f)$ do problema $\left(P_{1}\right)$.

\section{D.2 Propriedades do operador solução}

Vejamos algumas propriedades do operador solução $S$.

(P.1) $S$ é linear, isto é,

$$
S(\lambda f+\gamma g)=\lambda S(f)+\gamma S(g), \quad \forall f, g \in L^{2}(\Omega) \text { e } \forall \lambda, \gamma \in \mathbb{R} .
$$

\section{Demonstração:}

Sejam $u=S(f)$ e $v=S(g)$. Por definição

$$
\left\{\begin{aligned}
-\Delta u & =f, \quad x \in \Omega, \\
u & =0, \quad x \in \partial \Omega,
\end{aligned}\right.
$$

e

$$
\left\{\begin{aligned}
-\Delta v & =g, \quad x \in \Omega, \\
v & =0, \quad x \in \partial \Omega .
\end{aligned}\right.
$$

Multiplicando os problemas acima por $\lambda$ e $\gamma$ obtemos

$$
\left\{\begin{aligned}
-\Delta(\lambda u) & =\lambda f, \quad x \in \Omega, \\
\lambda u & =0, \quad x \in \partial \Omega
\end{aligned}\right.
$$

e

$$
\left\{\begin{aligned}
-\Delta(\gamma v) & =\lambda g, \quad x \in \Omega \\
\gamma v & =0, \quad x \in \partial \Omega
\end{aligned}\right.
$$

o que implica,

$$
\left\{\begin{aligned}
-\Delta(\lambda u+\gamma v) & =(\lambda f+\gamma g), \quad x \in \Omega \\
\lambda u+\gamma v & =0, \quad x \in \partial \Omega
\end{aligned}\right.
$$


e, consequentemente,

$$
S(\lambda f+\gamma g)=\lambda u+\gamma v
$$

isto é,

$$
S(\lambda f+\gamma g)=\lambda S(f)+\gamma S(g), \quad \forall f, g \in L^{2}(\Omega) \text { e } \forall \lambda, \gamma \in \mathbb{R} .
$$

(P.2) $S$ é contínuo, ou seja, existe $M>0$ verificando

$$
\|S(f)\| \leq M\|f\|_{L^{2}(\Omega)}, \quad \forall f \in L^{2}(\Omega) .
$$

\section{Demonstração:}

Seja $u=S(f)$. Então,

$$
\left\{\begin{aligned}
-\Delta u & =f, \quad x \in \Omega, \\
u & =0, \quad x \in \partial \Omega,
\end{aligned}\right.
$$

implicando que

$$
\int_{\Omega} \nabla u \nabla \phi d x=\int_{\Omega} f \phi d x \forall \phi \in H_{0}^{1}(\Omega) .
$$

Escolhendo $\phi=u$, obtemos

$$
\int_{\Omega}|\nabla u|^{2} d x=\int_{\Omega} f u d x
$$

e assim,

$$
\|u\|_{H_{0}^{1}(\Omega)}^{2}=\int_{\Omega} f u d x
$$

de onde segue

$$
\|u\|_{H_{0}^{1}(\Omega)}^{2} \leq \int_{\Omega}|f||u| d x .
$$

Por Hölder tem-se

$$
\|u\|_{H_{0}^{1}(\Omega)}^{2} \leq\|f\|_{L^{2}(\Omega)}\|u\|_{L^{2}(\Omega)} .
$$

Da desigualdade de Poincaré obtemos

$$
\|u\|_{H_{0}^{1}(\Omega)}^{2} \leq C\|f\|_{L^{2}(\Omega)}\|u\|_{H_{0}^{1}(\Omega)} .
$$

Se $u \neq 0$, então

$$
\|u\|_{H_{0}^{1}(\Omega)} \leq C\|f\|_{L^{2}(\Omega)},
$$

isto é,

$$
\|S(f)\| \leq C\|f\|_{L^{2}(\Omega)}, \quad \forall f \in L^{2}(\Omega) .
$$

(P.3) $S$ pode ser visto de $L^{2}(\Omega)$ em $L^{2}(\Omega)$. Além disso, neste caso $S$ é linear e compacto.

\section{Demonstração:}

Observe que,

$$
S: L^{2}(\Omega) \longrightarrow H_{0}^{1}(\Omega)
$$


é uma aplicação contínua e como a imersão

$$
i: H_{0}^{1}(\Omega) \longrightarrow L^{2}(\Omega)
$$

é compacta, recordando que

(a) composição de aplições lineares é linear;

(b) composição de um operador contínuo com um compacto é compacto;

podemos concluir que o operador

$$
S: L^{2}(\Omega) \longrightarrow L^{2}(\Omega)
$$

é linear compacto.

(P.4) O operador $S: L^{2}(\Omega) \rightarrow L^{2}(\Omega)$ é auto-adjunto, ou seja,

$$
(S(f), g)_{L^{2}(\Omega)}=(f, S(g))_{L^{2}(\Omega)}
$$

onde, $(., .)_{L^{2}}$ é o produto interno de $L^{2}(\Omega)$.

\section{Demonstração:}

Sejam $u=S(f)$ e $v=S(g)$. Devemos mostrar a seguinte igualdade

$$
\int_{\Omega} u g d x=\int_{\Omega} v f d x
$$

Desde que $u=S(f)$ e $v=S(g)$ temos que

$$
\left\{\begin{aligned}
-\Delta u & =f, \quad x \in \Omega, \\
u & =0, \quad x \in \partial \Omega,
\end{aligned}\right.
$$

e

$$
\left\{\begin{array}{rll}
-\Delta v & =g, & x \in \Omega, \\
v & =0, & x \in \partial \Omega .
\end{array}\right.
$$

Portanto,

$$
\int_{\Omega} \nabla u \nabla \phi d x=\int_{\Omega} f \phi d x, \quad \forall \phi \in H_{0}^{1}(\Omega)
$$

e

$$
\int_{\Omega} \nabla v \nabla \varphi d x=\int_{\Omega} g \varphi d x, \quad \forall \varphi \in H_{0}^{1}(\Omega) .
$$

Fixe $\phi=v$ e $\varphi=u$, obtemos

$$
\int_{\Omega} \nabla v \nabla u d x=\int_{\Omega} f v d x
$$

e

$$
\int_{\Omega} \nabla v \nabla u d x=\int_{\Omega} g u d x
$$

de onde segue

$$
\int_{\Omega} u g d x=\int_{\Omega} v f d x
$$


isto é, $(S(f), g)_{L^{2}(\Omega)}=(f, S(g))_{L^{2}(\Omega)}$.

(P.5) $S(f)=0$ se, e somente se, $f=0$.

\section{Demonstração:}

Considere que $S(f)=u$. Então, $u \in H_{0}^{1}(\Omega)$ é solução do problema

$$
\left\{\begin{aligned}
-\Delta u & =f, \quad x \in \Omega, \\
u & =0, \quad x \in \partial \Omega,
\end{aligned}\right.
$$

ou seja,

$$
\int_{\Omega} \nabla u \nabla \phi d x=\int_{\Omega} f \phi d x, \quad \forall \phi \in H_{0}^{1}(\Omega)
$$

Se $f=0$, então

$$
\int_{\Omega} \nabla u \nabla \phi d x=0, \quad \forall \phi \in H_{0}^{1}(\Omega)
$$

Para $\phi=u$, temos

$$
\int_{\Omega}|\nabla u|^{2} d x=\|u\|_{H_{0}^{1}(\Omega)}^{2}=0 \Leftrightarrow u=0
$$

isto é,

$$
S(f)=0 .
$$

Supondo que $S(f)=0$, segue de (D.2) que

$$
\int_{\Omega} f \phi d x=0, \quad \forall \phi \in H_{0}^{1}(\Omega)
$$

e então $f=0$.

(P.6) $\lambda=0$ não é autovalor de $S$.

\section{Demonstração:}

Usando que $N(S)=\left\{\varphi \in L^{2}(\Omega) ; S(\varphi)=0\right\}$, por (P.5), temos

$$
S(\varphi)=0 \Leftrightarrow \varphi=0 .
$$

Logo, $N(S)=0$, mostrando a propriedade.

(P.6) $S$ é positivo, isto é,

$$
(S(f), f)_{L^{2}(\Omega)}>0, \quad \forall f \neq 0 .
$$

\section{Demonstração:}

Seja $S(f)=u$ com $u \neq 0$, então

$$
\left\{\begin{aligned}
-\Delta u & =f, \quad x \in \Omega, \\
u & =0, \quad x \in \partial \Omega,
\end{aligned}\right.
$$


o que implica,

$$
\int_{\Omega} \nabla u \nabla \phi d x=\int_{\Omega} f \phi d x, \forall \phi \in H_{0}^{1}(\Omega)
$$

Fixando $\phi=u$, segue

$$
0<\|u\|_{H_{0}^{1}(\Omega)}^{2}=\int_{\Omega} f u d x=\int_{\Omega} f S(f) d x=(S(f), f)_{L^{2}(\Omega)},
$$

mostrando que

$$
(S(f), f)_{L^{2}(\Omega)}>0, \quad \forall f \neq 0 .
$$

Provadas as propriedades anteriores, usando o teorema espectral para operadores compactos autoadjuntos (veja [3]) concluímos que:

Afirmação 1: O operador $S: L^{2}(\Omega) \longrightarrow L^{2}(\Omega)$ tem uma sequência de autovalores reais positivos $\left\{\mu_{n}\right\}$ verificando

$$
\mu_{1}>\mu_{2}>\ldots>\mu_{n}>\ldots>0
$$

com

$$
\lim _{n \rightarrow \infty} \mu_{n}=0
$$

Além disso, temos que:

Afirmação 2: Os autovalores de $S$ são os inversos dos autovalores de

$$
\left\{\begin{aligned}
-\Delta u & =\lambda u, \quad x \in \Omega, \\
u & =0, \quad x \in \partial \Omega .
\end{aligned}\right.
$$

De fato, se $\lambda$ é um autovalor de $-\Delta$, então existe $\phi \neq 0$ verificando

$$
\left\{\begin{aligned}
-\Delta \phi & =\lambda \phi, \quad x \in \Omega, \\
\phi & =0, \quad x \in \partial \Omega .
\end{aligned}\right.
$$

Desde que $\lambda \neq 0$, segue da definição de solução fraca que

$$
\int_{\Omega} \nabla \phi \nabla \psi d x=\int_{\Omega} \lambda \psi \phi d x, \quad \forall \psi \in H_{0}^{1}(\Omega) .
$$

Fazendo $\phi=\psi$, temos

$$
\|\phi\|_{H_{0}^{1}(\Omega)}^{2}=\lambda\|\phi\|_{L^{2}(\Omega)}^{2},
$$

e consequentemente

$$
\lambda=\frac{\|\phi\|_{H_{0}^{1}}^{2}}{\|\phi\|_{L^{2}}^{2}}>0 .
$$

Considerando agora o problema

$$
\left\{\begin{aligned}
-\Delta\left(\frac{1}{\lambda} \phi\right) & =\phi,, \quad x \in \Omega, \\
\phi & =0, \quad x \in \partial \Omega,
\end{aligned}\right.
$$


temos

$$
S(\phi)=\frac{1}{\lambda} \phi
$$

mostrando que $\frac{1}{\lambda}$ é autovalor de $S$. Agora, se $\mu>0$ é autovalor de $S$, então existe $\phi \in L^{2}(\Omega)$, $\phi \neq 0, \mathrm{com}$

$$
S(\phi)=\mu \phi
$$

Logo,

$$
\frac{1}{\mu} S(\phi)=\phi
$$

Usando o fato que $S$ é linear, temos

$$
S\left(\frac{1}{\mu} \phi\right)=\phi
$$

de onde concluímos que

$$
\left\{\begin{aligned}
-\Delta \phi & =\frac{1}{\mu} \phi, \quad x \in \Omega \\
\phi & =0, \quad x \in \partial \Omega
\end{aligned}\right.
$$

implicando que $\frac{1}{\mu}$ é autovalor de $\left(P_{\lambda}\right)$.

Das Afirmações 1 e 2 concluímos que o problema $\left(P_{\lambda}\right)$ possui uma sequência $\left\{\lambda_{n}\right\}$ de autovalores reais positivos verificando

$$
0<\lambda_{1}<\lambda_{2}<\ldots<\lambda_{n}<\ldots
$$

e

$$
\lim _{n \rightarrow \infty} \lambda_{n}=\infty
$$

\section{D.3 Caracterização de $\lambda_{1}$}

Teorema D.3.1. Defina, para $v \in H_{0}^{1}(\Omega), v \neq 0$, o quociente de Rayleight

$$
R(v)=\frac{\|v\|_{H_{0}^{1}(\Omega)}^{2}}{\|v\|_{L^{2}(\Omega)}}=\frac{\int_{\Omega}|\nabla v|^{2} d x}{\int_{\Omega} v^{2} d x} .
$$

Então,

$$
\lambda_{1}=\min _{v \in H_{0}^{1}(\Omega) \text { e } v \neq 0} R(v)=R\left(w_{1}\right)
$$

e

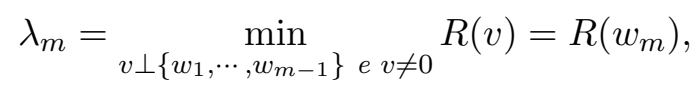

onde $w_{m}$ é a autofunção normalizada associada ao autovalor $\lambda_{j}$, para todo $j \in \mathbb{N}$. Além disso, $\left\{w_{n}\right\}$ é uma base em $H_{0}^{1}(\Omega)$. 


\section{Demonstração:}

Seja $W_{k}=\operatorname{span}\left\{w_{1}, \cdots, w_{k}\right\}$. É fácil ver que, para $m \geq 1, \lambda_{m}=R\left(w_{m}\right)$. Se $v \perp\left\{w_{1}, \cdots, w_{m-1}\right\}$, então a expansão de Fourier em $L^{2}(\Omega)$ de $v$ é dada por

$$
v=\sum_{k=m}^{\infty} \alpha_{k} w_{k}, \alpha_{k}=\int_{\Omega} v w_{k} d x
$$

Se tomarmos $v_{l}=\sum_{k=m}^{l} \alpha_{k} w_{k}$, então $v_{l} \rightarrow v$ em $L^{2}(\Omega)$ e em $H_{0}^{1}(\Omega)$ e, pela expansão de Fourier em $H_{0}^{1}(\Omega)$, temos

$$
v=\sum_{k=m}^{\infty} \lambda_{k}^{-\frac{1}{2}} \beta_{k} w_{k}
$$

com

$$
\beta_{k}=\lambda_{k}^{-\frac{1}{2}} \int_{\Omega} \nabla v \nabla w_{k} d x=\lambda_{k}^{\frac{1}{2}} \int_{\Omega} v w_{k} d x=\lambda_{k} \frac{1}{2} \alpha_{k} .
$$

Assim, $R\left(v_{l}\right) \rightarrow R(v)$. Agora, temos:

$$
R\left(v_{l}\right)=\frac{\sum_{k=m}^{l} \alpha_{k}^{2} \int_{\Omega} \nabla w_{k} \nabla w_{k} d x}{\sum_{k=m}^{l} \alpha_{k}^{2}}=\frac{\sum_{k=m}^{l} \alpha_{k}^{2} \lambda_{k}}{\sum_{k=m}^{l} \alpha_{k}^{2}} \geq \lambda_{m} .
$$

Assim, $R(v) \geq \lambda_{m}$, o que implica que a equação (D.5) é verdadeira. A equação (D.4) pode ser provada tomando-se $m=1$.

Mostremos que $\left\{w_{n}\right\}$ é base em $H_{0}^{1}(\Omega)$. Notemos que se $\left(u, w_{n}\right)_{H_{0}^{1}(\Omega)}=0, \forall m$, então $u \equiv 0$. De fato,

$$
\left(u, w_{n}\right)_{H_{0}^{1}(\Omega)}=\int_{\Omega} \nabla u \nabla w_{m} d x=\lambda_{m} \int_{\Omega} u w_{m} d x .
$$

Deste modo, $0=\left(u, w_{n}\right)_{H_{0}^{1}(\Omega)}=\lambda_{m}\left(u, w_{n}\right)_{L^{2}(\Omega)}$, implicando que $\left(u, w_{n}\right)_{L^{2}(\Omega)}=0, \forall m$, e assim, $u \equiv 0$

Logo, $\left\{w_{m}\right\}$ é completo em $H_{0}^{1}(\Omega)$, e portanto, $\left\{w_{m}\right\}$ também será uma base em $H_{0}^{1}(\Omega)$.

Lema D.3.1. Seja $w \neq 0$ em $H_{0}^{1}(\Omega)$ satisfazendo a relação $R(w)=\lambda_{1}$. Então, $w$ é uma autofunção correspondente $a \lambda_{1}$.

\section{Demonstração:}

Seja $v \in H_{0}^{1}(\Omega)$ e seja $t>0$. Então $w+t v \in H_{0}^{1}(\Omega)$, e, por (D.4),

$$
R(w+t v) \geq \lambda_{1}=R(w) .
$$

Sem perda de generalidade, podemos supor que $\|w\|_{H_{0}^{1}(\Omega)}^{2}=1$. Assim,

$$
\frac{\int_{\Omega} \nabla(w+t v) \nabla(w+t v) d x}{\int_{\Omega}(w+t v)^{2} d x} \geq \int_{\Omega} \nabla w \nabla w=\lambda_{1} d x .
$$

Portanto,

$$
\int_{\Omega} \nabla(w+t v) \nabla(w+t v) d x \geq \lambda_{1} \int_{\Omega}\left(w^{2}+2 t w v+t^{2} v^{2}\right) d x
$$


ou seja,

$$
\int_{\Omega}\left[(\nabla w)^{2}+t \nabla w \nabla v+t \nabla v \nabla w+t^{2} \nabla v \nabla v\right] d x \geq \lambda_{1}\left(\int_{\Omega} w^{2} d x+2 t \int_{\Omega} w v d x+t^{2} \int_{\Omega} v^{2} d x\right) .
$$

Pela desigualdade de Poincaré,

$\lambda_{1} \int_{\Omega} w^{2} d x+2 t \int_{\Omega} \nabla w \nabla v d x+t^{2} \int_{\Omega} \nabla v \nabla v d x \geq \lambda_{1} \int_{\Omega} w^{2} d x+\lambda_{1}\left(2 t \int_{\Omega} v w d x+t^{2} \int_{\Omega} v^{2} d x\right)$.

Portanto,

$$
t^{2} \int_{\Omega} \nabla v \nabla v d x+2 t \int_{\Omega} \nabla w \nabla v d x \geq \lambda_{1}\left(2 t \int_{\Omega} v w d x+t^{2} \int_{\Omega} v^{2} d x\right) .
$$

Dividindo a equação acima por $2 t$ e fazendo $t \rightarrow 0^{+}$, obtemos

$$
\int_{\Omega} \nabla w \nabla v d x \geq \lambda_{1} \int_{\Omega} v w d x
$$

Mas, fazendo $t \rightarrow 0^{-}$, obtemos:

$$
\int_{\Omega} \nabla w \nabla v d x \leq \lambda_{1} \int_{\Omega} v w d x
$$

Assim,

$$
\int_{\Omega} \nabla w \nabla v d x=\lambda_{1} \int_{\Omega} v w d x
$$

E, uma vez que $v \in H_{0}^{1}(\Omega)$ é arbitrário, segue que $w$ é autofunção correspondente a $\lambda_{1}$.

Teorema D.3.2. O primeiro autovalor $\lambda_{1}$ é simples e a correspondente autofunção não troca de sinal em $\Omega$

\section{Demonstração:}

Seja $w$ a autofunção correspondente a $\lambda_{1}$. Escrevemos $w=w^{+}-w^{-}$. Como $w^{+}$e $w^{-}$ estão em $H_{0}^{1}(\Omega)$, temos

$$
\int_{\Omega} \nabla w \nabla w^{+} d x=\lambda_{1} \int_{\Omega} w w^{+} d x
$$

Assim,

$$
\int_{\Omega} \nabla w^{+} \nabla w^{+} d x=\lambda_{1} \int_{\Omega}\left(w^{+}\right)^{2} d x
$$

e,

$$
\int_{\Omega} \nabla w^{-} \nabla w^{-} d x=\lambda_{1} \int_{\Omega}\left(w^{-}\right)^{2} d x .
$$

Agora, suponhamos que $w$ não possui sinal constante em $\Omega$. Neste caso, $w^{+}$e $w^{-}$não são identicamente nulas. Deduzimos então de (D.6) e (D.7) que

$$
R\left(w^{+}\right)=\lambda_{1}=R\left(w^{-}\right)
$$

Pelo Lema anterior $w^{+}$e $w^{-}$são autofunções de $\lambda_{1}$, isto é,

$$
-\Delta w^{+}=\lambda_{1} w^{+} \quad \text { e } \quad-\Delta w^{-}=\lambda_{1} w^{-} .
$$


Mas, como $w^{+}, w^{-} \geq 0$ pelo Teorema B.0.6, $w^{+}>0$ e $w^{-}>0$, pois nenhum das duas é identicamente nula. Tal fato é impossível, uma vez que $w^{+}$deve se anular quando $w^{-} \neq 0$ e vice-versa. Deste modo, $w$ possui sinal constante e é não-nula em $\Omega$. Portanto, podemos escolher uma $w_{1}>0$ em $\Omega$ que é uma autofunção correspondente é $\lambda_{1}$.

Suponhamos agora que $\lambda_{1}$ não seja simples. Assim, existiria outra autofunção ortogonal a $w_{1}$, digamos $\tilde{w}_{1}$. Deste modo,

$$
0=\int_{\Omega} \nabla w_{1} \nabla \tilde{w}_{1} d x=\int_{\Omega} w_{1} \tilde{w}_{1} d x
$$

o que é uma contradição, pois $w_{1}$ e $\tilde{w}_{1}$ tem sinal constante. Logo, $\lambda_{1}$ é um autovalor simples. 


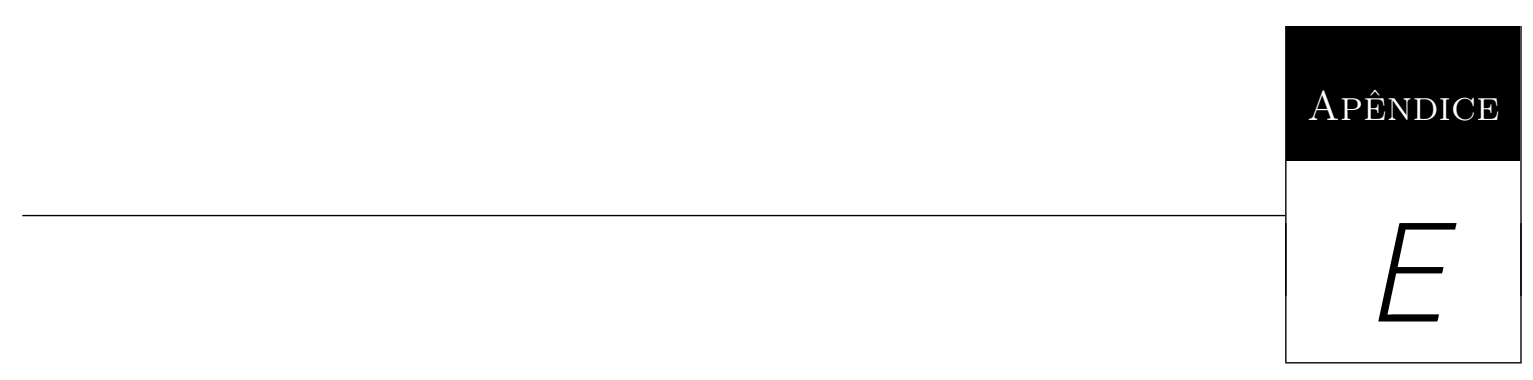

\section{Alguns resultados sobre Teoria do \\ Grau}

As demonstrações dos teoremas enunciados neste apêndice podem ser encontrados em [2]

\section{E.1 Grau de Brouwer}

\section{E.1.1 Caso Regular}

Definição E.1.1. Seja $S=\left\{x \in \Omega ; J_{f}[x]=0\right\}$, onde $J_{f}[x]=\operatorname{det}\left[f^{\prime}(x)\right]$.

Sejam $\Omega \subset \mathbb{R}^{N}$ um dominio limitado, $\varphi: \bar{\Omega} \rightarrow \mathbb{R}^{N}$ uma função de classe $\mathcal{C}^{1}(\bar{\Omega})$ e $b \notin \varphi(\partial \Omega) \cup \varphi(S)$. O Grau de Brouwer de $\varphi$ em $\Omega$ relacionado a b, denotado por $d(\varphi, \Omega, b)$ é dado por

$$
d(\varphi, \Omega, b)=\left\{\begin{array}{cl}
0, & \text { se } \quad b \notin \varphi(\bar{\Omega}), \\
\sum_{i=1}^{N} \operatorname{sgn}\left[J_{\varphi}\left[\xi_{i}\right]\right], & \text { se } \quad \varphi^{-1}(b)=\left\{\xi_{1}, \cdots, \xi_{N}\right\}
\end{array}\right.
$$

onde sgn $: \mathbb{R} \backslash\{0\} \rightarrow\{-1,1\}$ é dada por $\operatorname{sgn}(t)=\left\{\begin{aligned} 1, & \text { se } t>0, \\ -1, & \text { se } t<0 .\end{aligned}\right.$ 
Lema E.1.1. Seja $\varphi \in \mathcal{C}^{1}(\bar{\Omega})$ e $b \notin \varphi(\partial \Omega) \cup \varphi(S)$. Então existe uma vizinhança $U$ de $\varphi$ na topologia $\mathcal{C}^{1}$ tal que para todo $\psi \in U$, tem-se que:

(a) $b \notin \psi(\partial \Omega)$;

(b) Se $x \in \psi^{-1}(b)$, temos $J_{\psi}(x) \neq 0$; (isto é, $x \in \psi^{-1}(b) \Rightarrow x \notin S_{\psi}=\left\{x \in \bar{\Omega} ; J_{\psi}(x)=0\right\}$ );

(c) $d(\psi, \Omega, b)=d(\varphi, \Omega, b)$.

Corolário E.1.1. Seja $\varphi \in \mathcal{C}^{2}(\bar{\Omega})$ e $b_{1}, b_{2} \notin \varphi(\partial \Omega) \cup \varphi(S)$. Se $b_{1}$ e $b_{2}$ estiverem na mesma componente conexa de $\mathbb{R}^{N} \backslash \varphi(\partial \Omega)$, teremos $d\left(\varphi, \Omega, b_{1}\right)=d\left(\varphi, \Omega, b_{2}\right)$.

\section{E.1.2 Definição do Grau para $\varphi \in \mathcal{C}^{2}(\bar{\Omega})$ e $b \notin \varphi(\partial \Omega)$.}

Seja $\Omega \subset \mathbb{R}^{N}$ um aberto e limitado e $\varphi \in \mathcal{C}^{2}(\bar{\Omega})$. Se $b \notin \varphi(\partial \Omega)$, existe $x \in \mathcal{C}_{b} \backslash \varphi(S)$, onde $\mathcal{C}_{b}$ é a componente conexa de $\mathbb{R}^{N} \backslash \varphi(\partial \Omega)$, pois do contrário teríamos:

$$
\mathcal{C}_{b} \subset \varphi(S) \Rightarrow m(\varphi(S))>0,
$$

o que é um absurdo pelo Teorema de Sard.

Definição E.1.2. O grau de $\varphi$ em $\Omega$ com relação a b é dado por:

$$
d(\varphi, \Omega, b):=d(\varphi, \Omega, x) \text {, onde } x \in \mathcal{C}_{b} \backslash \varphi(S) .
$$

Note que o grau está bem definido, pois, se $x, y \in \mathcal{C}_{b} \backslash \varphi(S)$, pelo Corolário E.1.1 devemos ter $d(\varphi, \Omega, x)=d(\varphi, \Omega, y)$.

Corolário E.1.2. Seja $\varphi \in \mathcal{C}^{2}(\bar{\Omega})$ e $b \notin \varphi(\partial \Omega)$. Então existe uma vizinhança $\mathcal{U}$ de $\varphi$ em $\mathcal{C}^{2}(\bar{\Omega})$ induzida pela topologia $\mathcal{C}^{1}(\bar{\Omega})$ de maneira que se $\psi \in \mathcal{U}$, temos:

(i) $b \notin \psi(\partial \Omega)$;

(ii) $d(\varphi, \Omega, b)=d(\psi, \Omega, b)$.

Corolário E.1.3. Seja $H \in \mathcal{C}^{2}\left(\bar{\Omega} \times[0,1], \mathbb{R}^{N}\right) \operatorname{com} b \notin H(\partial \Omega \times[0,1])$. Então, $d(H(\cdot, t), \Omega, b) \equiv$ $C$ em $[0,1]$, onde $C$ é uma constante.

\section{E.1.3 Definição de Grau de Brouwer para funções contínuas.}

Seja $\Omega \subset \mathbb{R}^{N}$ um aberto limitado, $\varphi: \bar{\Omega} \rightarrow \mathbb{R}^{N}$ uma função contínua e $b \notin \varphi(\partial \Omega)$. Considere $r=\operatorname{dist}(b, \varphi(\partial \Omega))$. Sabemos do Teorema da Aproximação de Weierstrass que existe $\psi \in \mathcal{C}^{2}(\bar{\Omega})$, com

$$
\|\varphi-\psi\|_{\mathcal{C}(\bar{\Omega})}<\frac{r}{2}
$$

Para a definição do Grau de Brouwer para funções contínuas utilizaremos os dois Lemas a seguir: 
Lema E.1.2. Sejam $\psi_{1}, \psi_{2} \in \mathcal{C}^{2}(\bar{\Omega}) \operatorname{com}\left\|\varphi-\psi_{i}\right\|_{\mathcal{C}(\bar{\Omega})}<\frac{r}{2}, i=1,2$. Então $b \notin \psi_{i}(\partial \Omega)$, para $i=1,2 e$

$$
d\left(\psi_{1}, \Omega, b\right)=d\left(\psi_{2}, \Omega, b\right)
$$

Definição E.1.3. O grau de Brouwer de $\varphi$ em $\Omega$ com relação a $b$ como sendo $d(\varphi, \Omega, b):=$ $d(\psi, \Omega, b)$ para $\psi \in \mathcal{C}^{2}(\bar{\Omega})$ e $\|\varphi-\psi\|_{\mathcal{C}(\bar{\Omega})}<\frac{r}{2}$, onde $r=\operatorname{dist}(b, \varphi(\partial \Omega))$.

Lema E.1.3. Seja $\Omega \subset \mathbb{R}^{N}$ um domínio limitado, $\varphi: \bar{\Omega} \rightarrow \mathbb{R}^{N}$ contínua e $b \notin \varphi(\partial \Omega)$. Então, $d(\varphi, \Omega, b)=d(\varphi-b, \Omega, 0)$.

\section{E.1.4 Principais propriedades do Grau de Brouwer:}

1. Continuidade:

Seja $\varphi \in \mathcal{C}(\bar{\Omega})$ e $b \notin \varphi(\partial \Omega)$. Então existe uma vizinhança $\mathcal{U}$ de $\varphi$ em $\mathcal{C}(\bar{\Omega})$ de forma que:

(a) $b \notin \psi(\partial \Omega), \forall \psi \in \mathcal{U}$;

(b) $d(\varphi, \Omega, b)=d(\psi, \Omega, b), \forall \psi \in \mathcal{U}$.

2. Invariância do Grau por Homotopia Contínua:

Seja $H \in \mathcal{C}(\bar{\Omega} \times[0,1]) \operatorname{com} b \notin H(\partial \Omega \times[0,1])$. Então $d(H(\cdot, t), \Omega, b)$ é constante em $[0,1]$.

3. O grau é constante em componentes conexas:

Se $b$ e $b_{1}$ estão na mesma componente conexa de $\mathbb{R}^{N} \backslash \varphi(\partial \Omega)$, temos $d(\varphi, \Omega, b)=$ $d\left(\varphi, \Omega, b_{1}\right)$.

4. Aditividade do grau:

Seja $\varphi \in \mathcal{C}(\bar{\Omega})$ e $\Omega=\Omega_{1} \bigcup \Omega_{2}$ com $\Omega_{1}$ e $\Omega_{2}$ abertos, limitados e disjuntos. Seja $b \notin \varphi\left(\partial \Omega_{1}\right) \cup \varphi\left(\partial \Omega_{2}\right)$. Então $d(\varphi, \Omega, b)=d\left(\varphi, \Omega_{1}, b\right)+d\left(\varphi, \Omega_{2}, b\right)$.

\section{E.1.5 Consequências das Propriedades Principais}

No que segue $\Omega \subset \mathbb{R}^{N}$ é um conjunto limitado.

1. $d(I d, \Omega, b)= \begin{cases}1, & \text { se } b \in \Omega, \\ 0, & \text { se } b \notin \bar{\Omega} .\end{cases}$

2. Seja $\varphi: \bar{\Omega} \rightarrow \mathbb{R}^{N}$ uma função contínua $\operatorname{com} b \notin \varphi(\bar{\Omega})$. Então, $d(\varphi, \Omega, b)=0$.

3. Se $d(\varphi, \Omega, b) \neq 0$, então existe $x_{0} \in \Omega$ tal que $\varphi\left(x_{0}\right)=b$.

4. Se $d(\varphi, \Omega, b) \neq 0$, então $\varphi(\Omega)$ é uma vizinhança aberta de $b$, isto é, existe $r>0$ tal que $B_{r}(b) \subset \varphi(\Omega)$.

5. Se $\varphi(\Omega)$ está contido em um subespaço próprio de $\mathbb{R}^{n}$, então $d(\varphi, \Omega, b)=0$. 
6. Propriedade da exclusão: Seja $K \subset \bar{\Omega}$ um fechado com $b \notin \varphi(K)$. Então, $d(\varphi, \Omega, b)=$ $d(\varphi, \Omega \backslash K, b)$.

7. Seja $\left\{\Omega_{i}\right\}_{i \in I}$ uma família de abertos contidos em $\Omega$, dois a dois disjuntos com

$$
\varphi^{-1}(b) \subset \bigcup_{i \in I} \Omega_{i}
$$

Então, $d\left(\varphi, \Omega_{i}, b\right)=0$, a menos de um número finito de índices $i \in I \mathrm{e}$

$$
d(\varphi, \Omega, b)=\sum_{i \in I} d\left(\varphi, \Omega_{1}, b\right)
$$

8. Suponha que $\varphi=\psi$ em $\partial \Omega$ e que $\varphi$ e $\psi$ sejam funções contínuas. Então, para todo $b \notin \varphi(\partial \Omega)=\psi(\partial \Omega)$, temos $d(\varphi, \Omega, b)=d(\psi, \Omega, b)$.

\section{E.1.6 Índice de Brouwer}

Sejam $\Omega \subset \mathbb{R}^{N}$ um aberto limitado, $\varphi: \bar{\Omega} \rightarrow \mathbb{R}^{N}$ uma função contínua e $b \in \mathbb{R}^{N}$. Suponhamos que exista $x_{0} \in \Omega$ solução isolada da equação $\varphi(x)=b$. Assim, existe $r>0$ tal que a equação

$$
\varphi(x)=b, \quad \text { com } \quad x \in B_{r}\left(x_{0}\right),
$$

tem $x_{0}$ como única solução. Neste caso definimos o índice de Brouwer de $\varphi$ em $x_{0}$ relacionado a $b$ e denotado por $i\left(\varphi, x_{0}, b\right)$ como sendo

$$
i\left(\varphi, x_{0}, b\right)=d\left(\varphi, B_{r}\left(x_{0}\right), b\right)
$$

Usando a propriedade da exclusão, temos

$$
i\left(\varphi, x_{0}, b\right)=d\left(\varphi, B_{r}\left(x_{0}\right) \backslash K, b\right)
$$

onde $K=\overline{B_{r}\left(x_{0}\right)}-B_{\varepsilon}\left(x_{0}\right)$, isto é, $i\left(\varphi, x_{0}, b\right)=d\left(\varphi, B_{\varepsilon}\left(x_{0}\right), b\right)$, para todo $\varepsilon>0$ suficientemente pequeno. Deste modo a definição pode ser dada por

$$
i\left(\varphi, x_{0}, b\right)=\lim _{\varepsilon \rightarrow 0} d\left(\varphi, B_{\varepsilon}\left(x_{0}\right), b\right)
$$

Teorema E.1.1. Seja $\Omega$ um aberto limitado do $\mathbb{R}^{N}$ contendo a origem e $\varphi: \bar{\Omega} \rightarrow \mathbb{R}^{N}$ uma função da forma

$$
\varphi(x)=x-T x,
$$

onde $T: \mathbb{R}^{N} \rightarrow \mathbb{R}^{N}$ é uma função de classe $\mathcal{C}^{1}(\bar{\Omega})$ com $T(0)=0$. Se $J_{\varphi}[0] \neq 0$, temos

$$
i(\varphi, 0,0)=(-1)^{\beta}
$$

onde $\beta$ denota a soma das multiplicidades algébricas dos valores característicos de $T^{\prime}(0)$ contidos em $(0,1)$. 


\section{E.2 O Grau de Leray-Schauder}

Seja $E$ um espaço de Banach munido de uma norma $\|\cdot\|$ e seja $\rho(A, B)$ a distância entre conjuntos $A, B \subset E$.

Definição E.2.1. Seja $\Omega \subset E$ um aberto limitado. Diremos que uma aplicação da forma

$$
\begin{aligned}
& \phi(x): \bar{\Omega} \subset E \quad \rightarrow \quad E \\
& x \mapsto x-T(x)
\end{aligned}
$$

é uma perturbação de dimensão finita da identidade quando $T(\bar{\Omega})$ está contido em um subespaço de dimensão finita de $E$.

Definição E.2.2. Seja $\phi: \bar{\Omega} \subset E \rightarrow E \operatorname{com} \phi=I-T$ uma perturbação de dimensão finita da identidade, com $\Omega \subset E$ um aberto limitado. Se $b \notin \phi(\partial \Omega)$, definimos o grau de $\phi$ em $\Omega$ com relação a b como

$$
d(\phi, \Omega, b)=d\left(\left.\phi\right|_{\bar{\Omega} \cap F}, \Omega \cap F, b\right),
$$

onde $F$ é um subespaço de dimensão finita contendo $b$ e $T(\bar{\Omega})$.

Vejamos que o grau está bem-definido. Sejam $F_{1}$ e $F_{2}$ subespaços de $E$ com dimensão finita contendo $b$ e $T(\bar{\Omega})$. Note que o espaço $F=F_{1} \cap F_{2}$ é um subespaço de $E$ com dimensão finita que contém $b$ e $T(\bar{\Omega})$. Assim,

$$
d\left(\left.\phi\right|_{\bar{\Omega} \cap F_{1}}, \Omega \cap F_{1}, b\right)=d\left(\left.\phi\right|_{\bar{\Omega} \cap F_{1} \cap F}, \Omega \cap F_{1} \cap F, b\right)=d\left(\left.\phi\right|_{\bar{\Omega} \cap F}, \Omega \cap F, b\right) .
$$

De modo análogo,

$$
d\left(\left.\phi\right|_{\bar{\Omega} \cap F_{2}}, \Omega \cap F_{2}, b\right)=d\left(\left.\phi\right|_{\bar{\Omega} \cap F}, \Omega \cap F, b\right) .
$$

Portanto, a definição do grau independe da escolha de $F$.

Definição E.2.3. Sejam $(E,\|\cdot\|)$ um espaço vetorial e $\Omega \subset E$ um aberto limitado. Diremos que $T$ é uma aplicação compacta quando:

1. Té contínuo;

2. $T(\bar{\Omega})$ é relativamente compacto em $E$.

Observação E.2.1. Uma aplicação $T: E \rightarrow E$ é dita compacta quando $T$ é contínua e para qualquer limitado $U \subset E, T(U)$ é relativamente compacto.

Lema E.2.1. Seja $K \subset E$ um compacto. Então dado $\varepsilon>0$, existe um subespaço de dimensão finita $F_{\varepsilon}$ e uma função contínua $g_{\varepsilon}: K \rightarrow F_{\varepsilon}$ tal que $\left\|x-g_{\varepsilon}(x)\right\|<\varepsilon, \forall x \in K$.

Definição E.2.4. Quando $T: \bar{\Omega} \subset E \rightarrow E$ é um operador compacto, dizemos que a aplicação $\phi=I-T$ é uma perturbação compacta da identidade. 
Definição E.2.5. Uma aplicação é dita própria quando imagem inversa de compacto é um conjunto compacto.

Lema E.2.2. Seja $\phi=I-T: \bar{\Omega} \rightarrow E$ uma perturbação compacta da identidade. Então $\phi e ́$ uma aplicação fechada e própria.

Nosso objetivo é definir o grau para uma aplicação $\phi=I-T$ que é uma perturbação compacta da identidade. Seja $b \notin \phi(\partial \Omega)$. Desde que $\phi(\partial \Omega)$ é um fechado, pois $\phi$ é fechada, temos $0<r=\rho(b, \phi(\partial \Omega))$. Seja $K=\overline{T(\bar{\Omega})}$. Sendo $T$ um operador compacto, temos que $K$ é um conjunto compacto em $E$. Aplicando o Lema E.2.1, com $\varepsilon=\frac{r}{2}$, existe um subespaço de dimensão finita $F_{\frac{r}{2}}$ e uma aplicação contínua $g_{\frac{r}{2}}: K \rightarrow F_{\frac{r}{2}}$ tais que

$$
\left\|y-g_{\frac{r}{2}}\right\|<\frac{r}{2}, \forall y \in K=\overline{T(\bar{\Omega})} .
$$

Assim,

$$
\left\|T x-g_{\frac{r}{2}}(T x)\right\|<\frac{r}{2}, \forall x \in \bar{\Omega} .
$$

Considere a seguinte perturbação de dimensão finita da identidade: $\phi_{r}:=I-T_{r}: \bar{\Omega} \rightarrow E$, onde $T_{r}=g_{\frac{r}{2}} \circ T: \bar{\Omega} \rightarrow F_{\frac{r}{2}}$. Notemos que:

1. $b \notin \phi_{r}(\partial \Omega)$.

De fato, para $x_{0} \in \partial \Omega$, temos:

$$
\begin{gathered}
\left\|b-\phi_{r}\left(x_{0}\right)\right\|=\left\|b-\phi\left(x_{0}\right)+\phi\left(x_{0}\right)-\phi_{r}\left(x_{0}\right)\right\| \geq\left\|b-\phi\left(x_{0}\right)\right\|-\left\|\phi\left(x_{0}\right)-\phi_{r}\left(x_{0}\right)\right\| \geq \\
\geq r-\left\|I\left(x_{0}\right)-T\left(x_{0}\right)-\left(I\left(x_{0}\right)-g_{\frac{r}{2}}\left(T\left(x_{0}\right)\right)\right)\right\|=r-\left\|g_{\frac{r}{2}}\left(T\left(x_{0}\right)\right)-T\left(x_{0}\right)\right\|>r-\frac{r}{2}=\frac{r}{2} .
\end{gathered}
$$

Portanto, $b \notin \phi_{r}(\partial \Omega)$.

2. $\left\|\phi-\phi_{r}\right\|_{\mathcal{C}(\bar{\Omega})}<\frac{r}{2}$.

De fato, pois para qualquer $x \in \bar{\Omega}$,

$$
\left\|\phi(x)-\phi_{r}(x)\right\|=\left\|g_{\frac{r}{2}(T x)-T x}\right\| \leq \frac{r}{2} .
$$

Logo, $\left\|\phi-\phi_{r}\right\|_{\mathcal{C}(\bar{\Omega})}=\sup _{x \in \bar{\Omega}}\left\|\phi(x)-\phi_{r}(x)\right\| \leq \frac{r}{2}$.

Portanto, $\left\|\phi-\phi_{r}\right\|_{\mathcal{C}(\bar{\Omega})} \leq \frac{r}{2}$.

Definição E.2.6. Seja $\phi=I-T: \bar{\Omega} \rightarrow E$ uma perturbação compacta da identidade e $b \notin \phi(\partial \Omega)$. Definimos o Grau de Leray-Schauder de $\phi$ em $\Omega$ relacionado a $b$ como sendo:

$$
d(\phi, \Omega, b)=d\left(\phi_{r}, \Omega, b\right)
$$

onde $\phi_{r}: \bar{\Omega} \rightarrow E$ é uma perturbação de dimensão finita da identidade verificando

$$
\left\|\phi-\phi_{r}\right\|_{\mathcal{C}(\bar{\Omega})} \leq \frac{r}{2}
$$


Observação E.2.2. $\quad$ 1. A classe de perturbações de dimensão finita da identidade que realiza (E.2) é não-vazia, pois a perturbaçãa $\phi_{r}=I-T_{r}=g_{\frac{r}{2}} \circ T$ cumpre tal condiçãa.

2. Vejamos que o grau de $\phi$ em $\Omega$ relacionado a b está bem definido. Sejam $\phi_{i}=I-T_{i}$ : $\bar{\Omega} \rightarrow E, i=1,2$, perturbações de dimensão finita da identidade com

$$
\left\|\phi-\phi_{i}\right\|<\frac{r}{2}, \quad i=1,2 .
$$

Afirmação: $d\left(\phi_{1}, \Omega, b\right)=d\left(\phi_{2}, \Omega, b\right)$.

Sejam $F_{i}, i=1,2$, subespaços de dimensão finita contendo $T_{i}(\bar{\Omega})$, isto é

$$
T_{1}(\bar{\Omega}) \subset F_{1} \quad \text { e } \quad T_{2}(\bar{\Omega}) \subset F_{2} .
$$

Considere $F$ um subespaço de dimensão finita, contendo $F_{1}+F_{2}$ e b.

Logo,

$$
T_{1}(\bar{\Omega}), T_{2}(\bar{\Omega}) \subset F \quad e \quad b \in F
$$

Sabemos, por definição, que

$$
d\left(\phi_{i}, \Omega, b\right)=d\left(\left.\phi_{i}\right|_{\bar{\Omega} \cap F}, \bar{\Omega} \cap F, b\right), i=1,2 .
$$

No que segue, consideremos $\tilde{\phi}_{1}=\left.\phi_{1}\right|_{\bar{\Omega} \cap F}, \tilde{\phi}_{2}=\left.\phi_{2}\right|_{\bar{\Omega} \cap F}: \bar{\Omega} \cap F \rightarrow F e$

$$
\begin{aligned}
H: \theta \times[0,1] & \rightarrow F \\
(x, t) & \mapsto H(x, t)=t \tilde{\phi}_{1}(x)+(1-t) \tilde{\phi}_{2}(x),
\end{aligned}
$$

onde $\theta=\bar{\Omega} \cap F$. Note que $b \notin H(\partial \theta \times[0,1])$, com $\partial \theta=\partial_{F}(\bar{\Omega} \cap F) \subset \partial \Omega$. De fato, pois para $x_{0} \in \partial \theta$ e $t \in[0,1]$, temos

$$
\begin{aligned}
\left\|b-H\left(x_{0}, t\right)\right\| & =\left\|b-\phi\left(x_{0}\right)+\phi\left(x_{0}\right)-H\left(x_{0}, t\right)\right\| \\
& \geq\left\|b-\phi\left(x_{0}\right)\right\|-\left\|\phi\left(x_{0}\right)-H\left(x_{0}, t\right)\right\| \\
& >r-\left\|\phi\left(x_{0}\right)-\left(t \tilde{\phi}_{1}(x)+(1-t) \tilde{\phi}_{2}(x)\right)+t \phi\left(x_{0}\right)-t \phi\left(x_{0}\right)\right\| \\
& =r-\left(t\left\|\phi\left(x_{0}\right)-\tilde{\phi}_{1}\left(x_{0}\right)\right\|+(1-t)\left\|\phi\left(x_{0}\right)-\tilde{\phi}_{2}\left(x_{0}\right)\right\|\right) \\
& \geq r-t \frac{r}{2}-(1-t) \frac{r}{2} \\
& =r-\frac{t r}{2}-\frac{r}{2}+\frac{r t}{2} \\
& =\frac{r}{2} .
\end{aligned}
$$

Portanto,

$$
\left\|b-\phi\left(x_{0}\right)\right\| \geq \frac{r}{2}>0
$$

mostrando que $b \notin H(\partial \theta \times[0,1])$.

Pela invariância do Grau de Brouwer por homotopia, temos $d(H(\cdot, 0), \theta, b)=$ $d(H(\cdot, 1), \theta, b)$. Logo, $d\left(\tilde{\phi}_{1}, \Omega \cap F, b\right)=d\left(\tilde{\phi}_{2}, \Omega \cap F, b\right)$, implicando que $d\left(\phi_{1}, \Omega, b\right)=$ $d\left(\phi_{2}, \Omega, b\right)$, como queríamos. 


\section{E.2.1 Principais Propriedades do Grau de Leray-Schauder}

1. Continuidade em relação ao operador $T$ :

Seja $\phi=I-T: \bar{\Omega} \rightarrow E$ uma perturbação compacta da identidade e $b \notin \phi(\partial \Omega)$. Então existe uma vizinhança $\mathcal{U}$ de $T$ em $K(\bar{\Omega}, E)$ tal que:

(a) $b \notin(I-\psi)(\partial \Omega), \forall \psi \in \mathcal{U}$;

(b) $d(\phi, \Omega, b)=d(I-\psi, \Omega, b), \forall \psi \in \mathcal{U}$.

2. Invariância do Grau por Homotopia:

Seja $T \in K(\bar{\Omega} \times[0,1], E)$ e $H(u, t)=u-T(u, t)$. Se $b \notin H(\partial \Omega \times[0,1])$, temos que $d(H(\cdot, t), \Omega, b)$ é constante em $[0,1]$.

3. Aditividade do Grau:

Seja $\Omega=\Omega_{1}+\Omega_{2}$, onde $\Omega_{1}$ e $\Omega_{2}$ são abertos, limitados e disjuntos, e $\phi=I-T: \bar{\Omega} \rightarrow E$ uma perturbação compacta da identidade. Se $b \notin \phi(\partial \Omega)$ e $b \notin \phi\left(\partial \Omega_{i}\right), i=1,2$, então

$$
d(\phi, \Omega, b)=d\left(\phi, \Omega_{1}, b\right)+d\left(\phi, \Omega_{2}, b\right) .
$$

\section{E.2.2 Consequência das Propriedades Principais}

1. Se $I: E \rightarrow E$ é a aplicação identidade, então:

$$
d(I, \Omega, b)= \begin{cases}1, & b \in \Omega, \\ 0, & b \notin \bar{\Omega} .\end{cases}
$$

2. Se $b \notin \phi(\bar{\Omega})$, então $d(\phi, \Omega, b)=0$.

3. Se $d(\phi, \Omega, b) \neq 0$, então existe $u_{0} \in \Omega$ tal que $\phi\left(u_{0}\right)=b$.

4. Se $d(\phi, \Omega, b) \neq 0$, então $\psi(\bar{\Omega})$ é uma vizinhança de $b$, isto é, existe $\delta>0$ tal que $B_{\delta}(b) \subset \phi(\Omega)$.

5. Se $\phi(\Omega)$ está contido em um subespaço próprio de $E$, devemos ter $d(\phi, \Omega, b)=0$, $\forall b \notin \phi(\partial \Omega)$.

6. (Propriedade da exclusão). Se $K$ é um subconjunto fechado de $\bar{\Omega}$ com $b \notin \phi(K)$, então $d(\phi, \Omega, b)=d(\phi, \Omega \backslash K, b)$.

7. Seja $\left\{\Omega_{i}\right\} i \in I$ uma família de abertos, dois a dois disjuntos e $\Omega=\cup_{i \in I} \Omega_{i}$. Se $b \in E$ é tal que $\phi^{-1}(b) \subset \cup_{i \in I} \Omega_{i}$, então $d\left(\phi, \Omega_{i}, b\right)=0$, a menos de uma quantidade finita de índices $i$. Além disso, $d(\phi, \Omega, b)=\sum_{i \in I} d\left(\phi, \Omega_{i}, b\right)$.

8. Seja $\psi=I-S$, com $S \in K(\bar{\Omega}, E)$ tal que $\psi(x)=\phi(x)$ em $\partial \Omega$. Se $b \notin \phi(\partial \Omega)$, temos $d(\phi, \Omega, b)=d(\psi, \Omega, b)$. 
9. Não existe $\phi=I-T: \overline{B_{1}(0)} \rightarrow \partial B_{1}(0)$ perturbação compacta da identidade com $\left.\phi\right|_{\partial B_{1}(0)}=\left.I d\right|_{\partial B_{1}(0)}$.

10. (Teorema de Borsuk em dimensão infinita) Seja $\Omega \ni 0$ um aberto limitado de $E$ simétrico com relação a origem. Se $T \in K(\bar{\Omega}, E)$ é ímpar em $\partial \Omega$ e $0 \notin \phi(\partial \Omega)$, onde $\phi=I-T$, então $d(\phi, \Omega, 0)$ é ímpar.

\section{E.2.3 Índice de Leray-Schauder}

Sejam $\phi=I-T$ uma perturbação compacta da identidade, $b \notin \phi(\partial \Omega)$ e $u_{0}$ uma solução isolada da equação $\phi(u)=b$. Então existe $r>0$ tal que a única solução de $\phi(u)=b$ em $\overline{B_{r}\left(u_{0}\right)}$ é o próprio $u_{0}$. Fixando-se $K=\overline{B_{r}\left(u_{0}\right)} \backslash B_{\varepsilon}\left(u_{0}\right)$ com $0<\varepsilon<r$, temos que

$$
d\left(\phi, B_{r}\left(u_{0}\right), b\right)=d\left(\phi, B_{\varepsilon}\left(u_{0}\right), b\right), \forall \varepsilon>0 .
$$

Definimos o índice de $\phi$ em $u_{0}$ com relação a $b$ como sendo

$$
i\left(\phi, u_{0}, b\right)=\lim _{\varepsilon \rightarrow 0} d\left(\phi, B_{\varepsilon}\left(u_{0}\right), b\right)
$$

Teorema E.2.1. Sejam $L: E \rightarrow E$ um operador linear compacto e $\phi=I-L$. Se 1 não é valor caraterístico de $L$, então

$$
i(\phi, 0,0)=(-1)^{\beta},
$$

onde $\beta$ é a soma das multiplicidades dos valores característicos de $L$ contidos em $(0,1)$.

\section{Demonstração:}

Observemos inicialmente que $\phi(0)=0$ e que 0 é uma solução isolada de $\phi(u)=0$. De fato, se existisse $u \neq 0$ tal que $u=L u$, então 1 seria valor característico de $L$. Deste modo, faz sentido falar no índice $i(\phi, 0,0)$. Sejam $\mu_{1}, \mu_{2}, \cdots, \mu_{p}$ os valores característicos de $L$ contidos em $(0,1)$ e sejam $\alpha_{1}, \cdots, \alpha_{p}$ tais que

$$
N_{i}=\operatorname{ker}\left(\mu_{i} L-I\right)^{\alpha_{1}} .
$$

Consideremos

$$
N=\bigoplus_{i=1}^{p} N_{i},
$$

o qual possui dimensão finita e, portanto, é fechado. Seja $F \subset E$ um subespaço tal que $E=N \oplus F$. Denotemos por $P: E \rightarrow N$ e $Q: E \rightarrow F$ as projeções, que são contínuas. Consequentemente as aplicações $L \circ P: E \rightarrow E$ e $L \circ Q: E \rightarrow E$ são compactas. Note também que $N$ é invariante por $L$, isto é, $L(N) \subset N$.

Consideraremos agora

$$
\begin{aligned}
H: \overline{B_{1}(0)} \times[0,1] & \rightarrow E \\
(u, t) & \mapsto H(u, t)=u-L(P u)-t L(Q u) .
\end{aligned}
$$


Note que a aplicação

$$
\begin{aligned}
T: \overline{B_{1}(0)} \times[0,1] & \rightarrow E \\
(u, t) & \mapsto T(u, t)=L(P u)+t L(Q u)
\end{aligned}
$$

é um operador compacto em $\overline{B_{1}(0)} \times[0,1]$, uma vez que as aplicação $L \circ P$ e $L \circ Q$ são compactas.

Afirmação 1: $0 \notin H\left(\partial B_{1}(0) \times[0,1]\right)$.

De fato, para justificar tal afirmativa, vamos considerar o operador

$$
S=\prod_{i=1}^{p}\left(\mu_{i} L-I\right)^{\alpha_{i}}: E \rightarrow E .
$$

Uma vez que $\left(\mu_{i} x-1\right)^{\alpha_{i}}$ são primos entre si, tem-se ker $S=N$. Assim,

$$
0=H(u, t) \Leftrightarrow u-L(P u)-L(Q u)=0 \Leftrightarrow P u+Q u-L(P u)-L(Q u)=0 .
$$

Portanto,

$$
0=H(u, t) \Leftrightarrow t L(Q u)-Q u=P u-L(P u) \in N,
$$

implicando que $t L(Q u)-Q u \in N$.

10 CASO: $t \neq \mu_{i}$, para $i=1, \cdots, p$.

Pela equação (E.3), temos que

$$
S(t L(Q u)-Q u)=S(P u-L(P u))=0 .
$$

Logo,

$$
S(t L(Q u)-Q u)=0
$$

o que implica que

$$
(t L-I)(S(Q u))=0,
$$

uma vez que $S \circ L=L \circ S$. Sendo $t \neq \mu_{i}, i=1, \cdots, p$, concluímos que

$$
S(Q u)=0 \Rightarrow Q u \in N \Rightarrow Q u \in N \cap F=\{0\} \Rightarrow Q u=0 .
$$

Logo, $u=P u+Q u=P u$ e assim,

$$
0=H(u, t) \Leftrightarrow u=L u
$$

implicando que 1 é valor característico associado a $u$, o que é um absurdo.

$\mathbf{2}^{\mathbf{o}}$ CASO: $t=\mu_{i}$, para algum $i=1, \cdots, p$.

Sabemos que $\left(\mu_{i} L-I\right) S(Q u)=0$, ou seja, $Q u \in \operatorname{ker}\left[\left(\mu_{i} L-I\right) \circ S\right]$. Pela definição de $S$,

$$
Q u \in \operatorname{ker}\left[\prod_{k=1, k \neq i}^{p}\left(\mu_{k} L-I\right)^{a_{k}} \circ\left(\mu_{i} L-I\right)^{\alpha_{i+1}}\right] \text {. }
$$


Logo,

$$
Q u \in \bigoplus_{i=1}^{p} \operatorname{ker}\left(\mu_{k} L-I\right)^{a_{k}} \bigoplus \operatorname{ker}\left(\mu_{i} L-I\right)^{\alpha_{i+1}}=\bigoplus_{i=1}^{p} N_{k} \bigoplus N_{i}=N,
$$

chegando a uma contradição como no $1^{\circ} \mathrm{CASO}$. Provamos assim a Afirmação 1. Usando a invariância do Grau por homotopia (propriedade (2) da seção E.2.1), temos

$$
d\left(\phi, \overline{B_{1}(0)}, 0\right)=d\left(H(\cdot, 0), \overline{B_{1}(0)}, 0\right)=d\left(H(\cdot, 1), \overline{B_{1}(0)}, 0\right)=d\left(I-L P u, \overline{B_{1}(0)}, 0\right) .
$$

E, assim

$$
i(\phi, 0,0)=d\left(\left.(I-L P)\right|_{N}, \overline{B_{1}(0)} \cap N, 0\right),
$$

ou seja,

$$
i(\phi, 0,0)=d\left(\left.(I-L)\right|_{N}, \overline{B_{1}(0)} \cap N, 0\right),
$$

implicando que

$$
i(\phi, 0,0)=i\left(\left.(I-L)\right|_{N}, 0,0\right)=(-1)^{\beta},
$$

pois trata-se do índice de Brouwer (Teorema E.1.1) e $\beta$ é a soma das multiplicidades dos valores característicos de $L$ contidos em $(0,1)$. 



\section{Referências Bibliográficas}

[1] BENCI, V., RABINOWITZ, P. H. Critical point theorems for indefinite functionals. Invent. Math., 52, p. 241-273, 1979.

[2] BERESTYCKI, H. Methodes Topologiques et problemes aux limites non lineares. 1975. Thèse de 3e cycle (Mathématiques pures). L'Universite de Paris VI, Paris, 1975.

[3] BREZIS, H. Analyse Fonctionnelle, Théorie et applications. Paris: Masson, 1983.

[4] CLARK, D. C. A variant of the Ljusternik-Schnirelmann theory. Indiana Univ. Math. J., 22, p. 65-74, 1972.

[5] DE FIGUEIREDO, D. G. Lectures on the Ekeland variational principle with applications and detours. Berlin New York: Published for the Tata Institue of Fundamental Research [by] Springer-Verlag, 1989.

[6] DE FIGUEIREDO, D. G. Um convite às equações elípticas. Não publicado.

[7] GILBARG, D., TRUDINGER, N. S. Elliptic partial differential equations of second order. 2ed. Berlin-New York: Springer-Verlag, 1998.

[8] KAVIAN, O. Introduction à la théorie des points critiques et applications aux problèmes elliptiques. Heidelberg: Springer, 1993.

[9] KESAVAN, S. Topics in Functional Analysis and Applications. New Delhi: Wiley Eastern Limited, 1989.

[10] PALAIS, R. S. Critical point theory and the minimax principle. Proc. Sympos. Pure Math., v. 15, p. 185-212, 1970.

[11] RABINOWITZ, P. H. Minimax methods in critical point theory with applications to differential equations. Providence: AMS, 1986. 
[12] RĂDULESCO,V. Remarks on a limiting case in the treatment of nonlinear problems with mountain pass geometry. Studia Univ. Babes-Bolyai, Mathematica, v. LV, n. 4, p. 99-106, 2010.

[13] STRUWE, M. Variational methods : applications to nonlinear partial differential equations and hamiltonion systems. Berlin: Springer, 1990.

[14] WILLEM, M. Minimax theorems. Boston: Birkhauser, 1996. 